\title{
Non-stationary shock motion unsteadiness in an axisymmetric geometry with pressure gradient
}

\author{
W. J. Baars · J. H. Ruf · C. E. Tinney
}

Received: date / Accepted: date

\begin{abstract}
Shock wave / boundary layer interaction is studied in a large area ratio axisymmetric nozzle comprising a design exit Mach number of 5.58. Shock motion unsteadiness is captured by way of the dynamic wall pressure and is evaluated during overexpanded operations up to a nozzle pressure ratio of 65 . Stationary SWBLI is first considered at a nozzle pressure ratio of 28.7 such that the internal flow structure is in a restricted-shock separated state; the mean position of the annular separation shock resides at a fixed position. Conditional averages of the wall pressure fluctuations show how the motion of the incipient separation shock is out of phase with pressure fluctuations measured in the separated region downstream of the shock; pressure decreases when the shock moves downstream and vice versa. This is indicative of a long intermittent region, in terms of the boundary layer thickness, as the observed phenomena can be explained by translating the static wall pressure profile along with the shock motion. Non-stationary SWBLI is then considered by increasing the nozzle pressure ratio over time (transient startup). Under these conditions, the shock pattern varies in strength and structure as it sweeps through the nozzle. A time-frequency analyses of the fluctuating wall
\end{abstract}

\section{W. J. Baars $(\varangle)$}

Department of Mechanical Engineering, The University of Melbourne, Parkville, Victoria 3010, Australia

e-mail: wbaars@unimelb.edu.au

J. H. Ruf

ER41/Propulsion Structural and Dynamics Analysis Branch, NASA MSFC, Huntsville, AL 35812, USA

C. E. Tinney

Department of Aerospace Engineering and Eng. Mechanics, The University of Texas at Austin, Austin, TX 78712, USA

The experimental campaign was conducted at The University of Texas at Austin during the Spring of 2012. pressure during the non-stationary operations, and at the same location that the stationary unsteadiness is analysed, reveals a similar spectral footprint. However, for relatively slower start-ups, the amplitude of the unsteadiness is reduced by a factor of about seven. The findings demonstrate how the rate at which the nozzle pressure ratio increases can have a significant influence on the amplitude of the unsteady shock foot motion.

\section{Introduction}

This work focusses on Shock Wave / Boundary Layer Interactions (SWBLI) that form within the divergent section of a convergent-divergent axisymmetric nozzle. These kinds of interactions commonly form in large area-ratio rocket nozzles during off-design operations of the engine (Babinsky and Harvey, 2011). Strong interactions lead to concentrated thermal loads, high intensity acoustic radiation, or even undesirable structural loads that act on the vehicle and its surrounding structure. Thus, a comprehensive understanding of the mechanisms responsible for generating this unsteadiness would eventually lead to the development of lighter and more robust launch vehicles or other high speed vehicles comprising complex geometries.

SWBLI type research topics are driven by a continued interest in trying to collectively understand (and accurately predict) turbulent boundary layer flows (Smits and Dussauge, 2006), shock wave dynamics, and multidisciplinary physics. A great many investigations have been conducted using an assortment of geometries including axisymmetric cones, compression ramps and protruding surface cylinders. Here we focus on developing a framework for studying both stationary and non-stationary SWBLIs that form in overexpanded and 
large area-ratio rocket nozzles. The platform for this investigation comprises a large area ratio thrust optimized parabolic contour (TOP) nozzle with a design exit Mach number of 5.58.

For some TOP rocket nozzles, two flow states can form inside the nozzle when the flow is overexpanded. That is, during the start transient prior to the nozzle flowing full, and during shutdown transient after the nozzle no longer flows full (Frey and Hagemann, 2000; Nguyen et al, 2003; Ruf et al, 2009; Baars et al, 2012; Verma and Haidn, 2014). The two flow states are Free Shock Separation (FSS) and Restricted Shock Separation (RSS). The first of these, FSS, occurs at low Nozzle Pressure Ratios $\left(\mathrm{NPR}=p_{0} / p_{\infty}\right)$, and is characterized by the formation of a single shock that incipiently separates the expanding supersonic flow from the wall. The shock and separated flow are triggered by a growing adverse pressure gradient that forms between the expanding flow and the subsonic entrainment region. As NPR increases, the flow abruptly transitions from a FSS state to a RSS state. During the latter, shockbounded flow regions form and take on the shape of annular separation bubbles; a schematic of this is shown in Fig. 1. Several SWBLIs occur along the expanding wall of the nozzle to form a complex annular shock train with a subsonic flow region residing along the nozzle axis and downstream of the Mach disk (which connects to a strong reflected shock). Shocks interact with either the incoming boundary layer or invoke an unsteady coupling with the shock-bounded separated flow. In general, during both FSS and RSS conditions, the unsteadiness of the shock system is driven by mechanisms that are typically found in classical SWBLI studies, as well as by mechanisms that are induced by highly overexpanded nozzle flows, such as unsteady recirculating flows that can by itself result in axial fore and aft movement of the shock train.

Studies that focus on internal SWBLI and shockinduced nozzle flow separation in large area ratio nozzles are sparse. Planar nozzle configurations are commonly found in the open literature, given the optical access afforded by having non-diverging walls. For example, Johnson and Papamoschou (2010) used a spark schlieren system coupled with dynamic wall and pitot probe pressure measurements to visualize shock motion unsteadiness in a planar nozzle, which was shown to increase in amplitude with increasing shock strength. Olson and Lele (2013) accompanied these measurements by constructing a large-eddy simulation of the same flow to show how this low-frequency shock motion unsteadiness was driven by transonic resonance (Zaman et al, 2002). What is common about many of these planar nozzle studies is that there is just one single sepa-
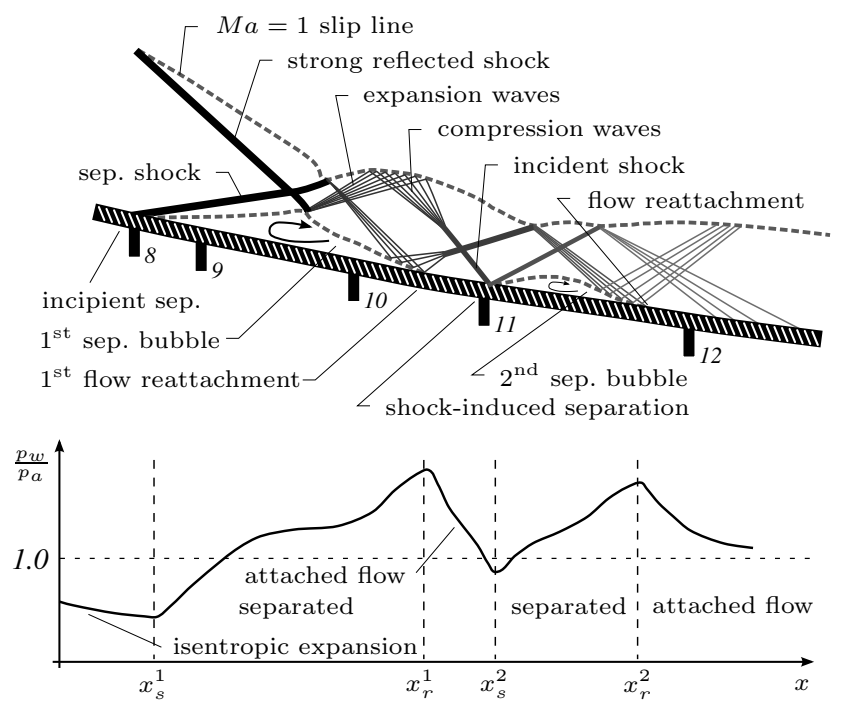

Fig. 1 Detailed overview of the RSS internal nozzle flow; a typical time-averaged wall pressure profile is indicated below, extracted from Baars et al (2012)

ration location, followed by a subsonic entrainment region. In this FSS flow state this subsonic separated flow region extends to the nozzle exit, and hence, is directly connected to the ambient fluid. Here we investigate SWBLI at the incipient separation shock that forms in an axisymmetric and large area ratio nozzle during a RSS state. Under these conditions, the downstream annular flow near the wall comprises both subsonic and supersonic flow and contains separation bubbles which are expected to suppress the mechanism responsible for the low-frequency shock motion unsteadiness associated with transonic resonance.

Given the complexity of SWBLI flows, an agreed upon set of parameters for a unique scaling of the unsteady phenomena are often difficult to accurately assess (given the limitations with current state-of-the-art experimental tools or high fidelity simulations). Even with simple two-dimensional compression ramps or flat plate geometries, significant discrepancies between the dominant low-frequency shock motion and the characteristic high-frequency content induced by the turbulent boundary layer have been observed. Plausible explanations continue to be a topic of scientific interest, a discussion of which an be found in the published reviews by Dolling (2001) and Clemens and Narayanaswamy (2014). Studies that consider the effect of the upstream flow on SWBLIs include the work of Beresh et al (2002), Ganapathisubramani et al (2009), Humble et al (2009) while the influence that the downstream flow might have on SWBLI unsteadiness is outlined in detail by Erengil and Dolling (1991), Thomas et al (1994), Dupont et al (2006), Piponniau et al (2009), Wu and Martín (2008), Priebe and Martín (2012), and the references therein. 
Nevertheless, where the characteristic frequency of the low-frequency shock motion unsteadiness is concerned, it is known to reside within a Strouhal number range of $S t_{L_{i}}=\left(f_{c}\right)_{\max } L_{i} / U_{\infty} \approx 0.01-0.03$ for flat plate, zero pressure gradient (ZPG) turbulent boundary layers (Gonsalez and Dolling, 1993). $L_{i}$ is defined as the length of the intermittent region and $\left(f_{c}\right)_{\max }$ is the maximum zero-crossing frequency (average number of shock foot passes per unit time) in the intermittent region. In this study, the dynamic wall pressure sensor spacing will prevent us from resolving the intermittent length scale. Furthermore, given the thin boundary layer that forms between the nozzle throat and the first separation shock (the thickness is the same order as the transducer diaphragm) it is not possible to resolve the full spectrum of scales associated with the incoming boundary layer fluctuations. This limits our ability to correlate the upstream flow with the low-frequency shock motion unsteadiness measured by way of high speed wall pressure sensors. Thus, because $L_{i}$ is not available, we will resort to the dimensionless expressions determined from the Mach 2 compression ramp study of Ganapathisubramani et al (2009). They show that the most energetic unsteadiness is in the band $f \delta_{0} / u_{e} \approx 0.008-0.025$; note that $\delta_{0}$, the incoming boundary layer thickness, is used as the length scale as opposed to the length of the intermittent region. Also, $u_{e}$ is the boundary layer edge velocity, which would equal the free-stream velocity $U_{\infty}$ in the case of external flows.

An outline of the manuscript is as follows. The experimental campaign is described in $\S 2$. Stationary nozzle operations are then used in $\S 3$ to examine the incipient separation shock, as well as various points along the wall downstream of this shock, during the RSS state. In $\S 4$, the nozzle is operated in a transient manner in order to elucidate non-stationary SWBLI. Non-stationary SWBLIs are more relevant to real rocket engine operations considering the transient start-up (nozzle filling) incurred during vehicle launch and the transient startups and shut-downs (nozzle emptying) as encountered during engine testing. Thus, the shock pattern varies in strength and structure when moving through the nozzle, and so, the unsteadiness is superposed on a nonstationary and transient mean flow.

\section{Experimental campaign}

\subsection{Hardware and instrumentation}

Experiments were conducted in the fully anechoic chamber and open-jet facility at the University of Texas at Austin (for details see Baars and Tinney, 2013). The axisymmetric TOP contoured nozzle (Ruf et al, 2010)

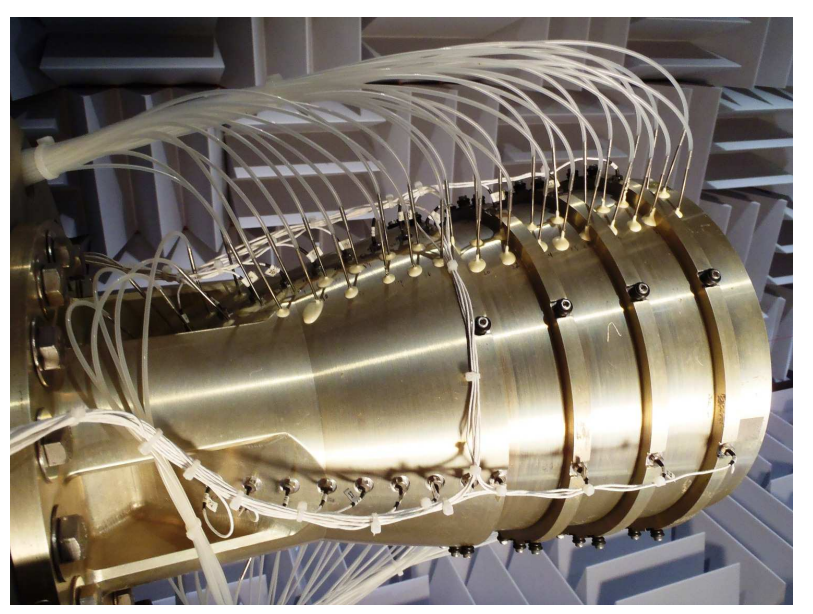

Fig. 2 Instrumented TOP nozzle installed in the fully anechoic chamber at The University of Texas at Austin

(a)

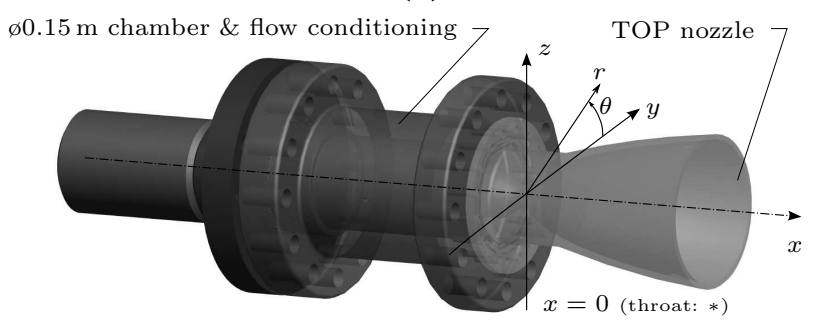

(b)
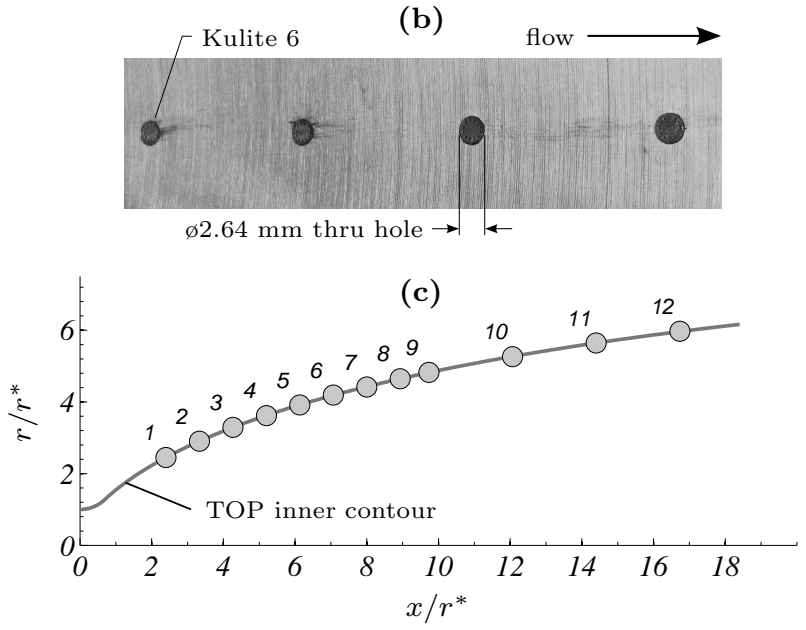

Fig. 3 a Schematic of the nozzle set-up with coordinate system. b Detailed photo of flush-mounted Kulite XT-140 transducers. c Locations of Kulites in the curvilinear array

shown in Fig. 2, served as the platform for studying SWBLIs inside axisymmetric geometries with strong FPG. The nozzle has a throat radius of $r^{*}=19.05 \mathrm{~mm}$, an exit-to-throat area-ratio of 38 (design exit Mach number of $\left.M_{d}=5.58\right)$, and an exit plane located at $x / r^{*}=18.40$. A schematic of the nozzle and plenum is shown in Fig. 3a, alongside a profile of the nozzle's interior surface in Fig. 3c. Static wall pressures were measured using 32 ports ( $\varnothing 1.10 \mathrm{~mm}$ thru hole) with an 
equidistant axial spacing of $\Delta x / r^{*}=0.47$. These ports alternated between two axial rows at $\theta=100^{\circ}$ and $\theta=110^{\circ}$, and spanned the range $x / r^{*}=[3.93,17.80]$, as is shown by the white pressure tubing in Fig. 2. Two Scanivalve DSA3218 modules with 50 psia ( $\pm .05 \%$ FS) range pressure sensors were connected to these 32 ports and recorded the static pressure at a rate of $440 \mathrm{~Hz}$.

Two curvilinear arrays of Kulite XT-140 dynamic pressure transducers were employed to sense the fluctuating wall pressure, which are identified by the white electric wiring in Fig. 2. The arrays were positioned at $\theta=90^{\circ}$ and $180^{\circ}$ and encompassed 12 transducers each; their locations are indicated in Fig. $3 \mathrm{c}$ and are further referred to as $x_{j}, j=1 \ldots 12$. These Kulite transducers comprise a dynamic range of 100 psia $( \pm 0.1 \%$ FS $)$ and were installed in the nozzle such that their protective Btype screens, with $2.62 \mathrm{~mm}$ outside diameter, were flush with the interior surface (Fig. 3b). These screens limit the effective frequency response up to $20 \mathrm{kHz}$. Therefore, all 24 channels were sampled simultaneously at a rate of $f_{s}=40 \mathrm{kHz}$ using a National Instruments PXI based system.

\subsection{Flow conditions}

Nozzle pressure ratios were regulated using a control valve located well upstream of the settling chamber and downstream of pressurized tanks that stored unheated air at a maximum pressure of $140 \mathrm{~atm}$. Ambient temperature, ambient pressure, total temperature inside the settling chamber, and relative humidity were recorded as $T_{\infty}=294.0 \mathrm{~K}, p_{\infty}=100.5 \mathrm{kPa}, T_{0}=$ $285 \mathrm{~K}$ and $\mathrm{RH}=32 \%$, respectively. Under these conditions, the velocity at the nozzle throat is valued at $a^{*}=308.9 \mathrm{~m} / \mathrm{s}$ using isentropic flow equations.

The analysis in $\S 3-4$ corresponds to two operating scenarios that govern a fixed NPR condition and a set of transient NPR conditions. The fixed NPR case provides insight into the wall pressure unsteadiness that forms during steady operation of the nozzle. The NPR operating curve of a typical experiment lasting $\sim 14$ seconds is shown in Fig. 4a. Only the time domain $t \in t_{s}$ is considered (highlighted in black) and has a steady operating condition of $\mathrm{NPR}=28.7$. In addition, the unsteady wall pressure signal at location $x_{8}$ exhibits features of shock crossings and shock motion unsteadiness due to it being located in the intermittent region of the initial separation shock. It is important to realize that the pressure floor of $p\left(x_{8}, t\right)$ corresponds to the wall pressure of the attached boundary layer (no transducer saturation) when the separation shock foot resides downstream of $x_{8}$; the pressure floor value is nominally proportional to the NPR. Since we focus on
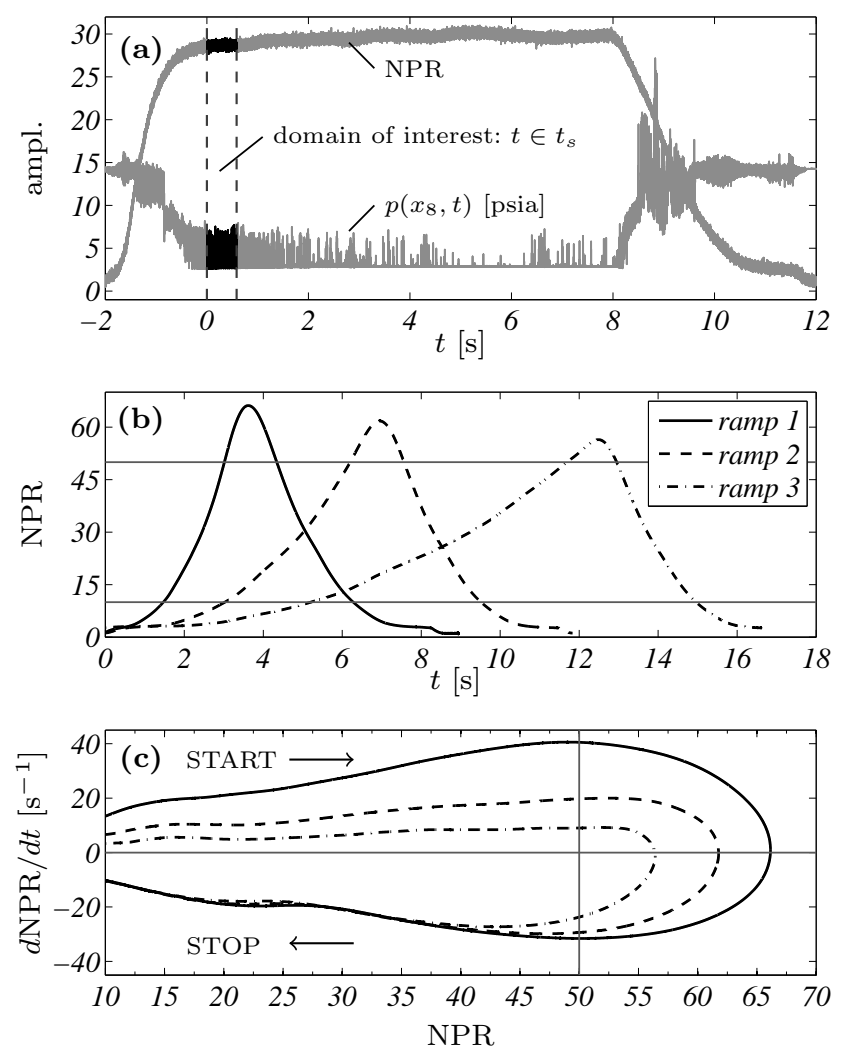

Fig. 4 a NPR trend and dynamic wall pressure during fixed NPR operation. b NPR curves during ramp 1 to 3 for startup \& shut-down. c Ramp rate as function of NPR for ramp 1 to 3 , a ramp rate of $d \mathrm{NPR} / d t=20 \mathrm{~s}^{-1}$ corresponds to a non-dimensional ramp rate of $\left(L / a^{*}\right) d \mathrm{NPR} / d t=2.3 \cdot 10^{-2}$

the shock unsteadiness, these variations affiliated with the operating conditions have been removed, and so, the current pressure floor has a value that corresponds to the mean NPR of 28.7. For transient conditions, three separate runs are considered which contain both startup and shut-down sequences. Figs. $4 \mathrm{~b}$ and $\mathrm{c}$ show the NPR trends of these three runs; denoted as ramps 1 to 3 . While shut-down rates are indistinguishable, startup rates were designed to be different and are lower for each subsequent ramp number.

\subsection{Boundary layer characteristics}

Several boundary layer parameters are of interest in this study as they provide a basic understanding of the incoming flow immediately above the surface where the SWBLI phenomenon is studied. Given the unavailability of flow measurements at this time, these parameters are provided by way of a steady Reynolds-averaged Navier-Stokes (RANS) model. This RANS computation utilized the Loci-Chem code (Luke and Cinella, 2007; Luke, 2007) with an axisymmetric and steady- 
(a)

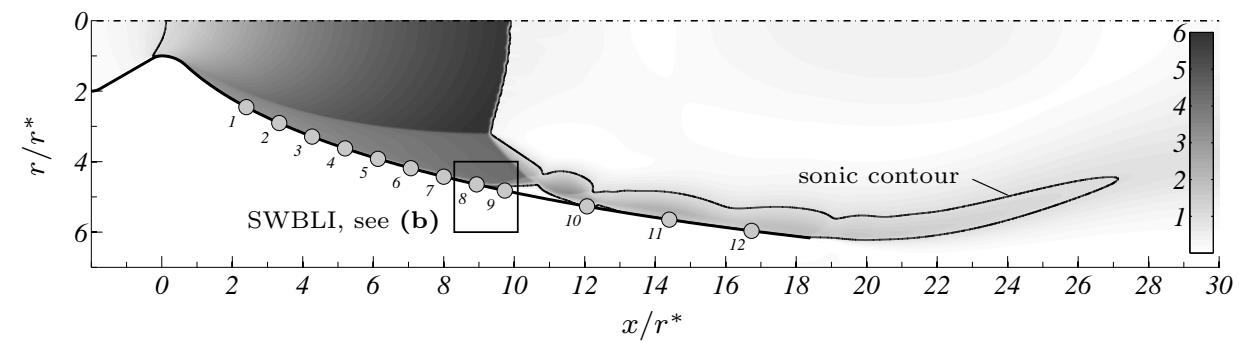

(b)

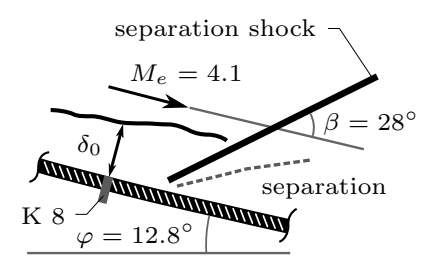

Fig. 5 a Mach number contour from the RANS model at NPR $=28.7$. b Detail of the SWBLI at the incipient separation

state analysis comprising unheated air as the fluid medium. of the local free-stream velocity, was extracted to proThe total pressure and temperature conditions were matched with the experimental scenario (see $\S 2.2$ ). The Menter baseline turbulence model (Menter, 1994) was implemented, which encompasses a blending of the $\kappa-\varepsilon$ and $\kappa-\omega$ models, as well as a compressibility correction (Sarkar and Lakshmanan, 1991). The computational mesh comprised $\sim 40$ cells within the viscous sublayer with the first cell residing at $y^{+} \approx 1$. Thus, no boundary layer characteristics were assumed a priori in this simulation. Wall pressure profiles from the simulation were shown to compare remarkably well to the measurements performed in the UT-Austin facility (see Donald et al, 2014). The RANS model was furthermore validated through a grid refinement study, which revealed unaltered separation locations of the internal flow, as well as unaffected boundary layer profiles upstream of the incipient separation shock. In addition, no significant change was observed in the boundary layer profile or the separation location when the baseline turbulence model was run with the Wilcox compressibility correction (Wilcox, 1998) (in place of the Sarkar compressibility correction) or if Menter's shear stress transport turbulence model (Menter, 1992) was implemented with either of those compressibility corrections. The steady-state RANS model constructed here was computed at NPR $=28.7$ in order to match the stationary, non-transient, experimental case. Mach number contours are shown in Fig. 5a and illustrate that the flow is in RSS state. The incipient separation shock resides around location $x_{8}$ and is consistent with the static wall pressure profiles measured in the laboratory (see Fig. 8 on subsequent pages) thereby providing additional confidence in this RANS simulation.

Wall-normal velocity profiles were extracted from the RANS model at 200 equally spaced axial locations, ranging from $x=0$ to $x=x_{8}$. Given the changing curvature of the wall, the local wall-tangential velocity was taken along wall-normal profiles using $u=\sqrt{u_{x}^{2}+u_{r}^{2}}$, where $u_{x}$ and $u_{r}$ are axial and radial velocities, respectively. The boundary layer thickness $\delta$, based on $99 \%$ duce the normalized growth profile shown in Fig. 6a. Subscripts 'e' and 'w' indicate conditions at the boundary layer edge and wall, respectively. Wall-normal profiles of velocity and Mach number at location $x_{8}$ are shown in Fig. 6b using inner-scaling on both axes. Velocity and the wall-normal coordinate are made dimensionless using $u^{+}=u\left(y^{+}\right) / u_{\tau}$ and $y^{+}=y^{\prime} u_{\tau} / \nu_{w}$, respectively, where $\nu_{w}=\mu_{w} / \rho_{w}=6.047 \cdot 10^{-5} \mathrm{~m}^{2} / \mathrm{s}$ and is the kinematic viscosity at the wall computed through Sutherland's law (local thermodynamic equilibrium) from the local wall temperature $T_{w}$. The friction velocity $u_{\tau}=\sqrt{\tau_{w} / \rho_{w}}$ is obtained by computing the skin-friction from the derivative of the linear velocity profile at the wall, e.g. $\tau_{w}=\mu_{w} \partial u / \partial y^{\prime}$. This resulted in a skin-friction valued at $\tau_{w}=206.6 \mathrm{~N} / \mathrm{m}^{2}$, a friction velocity of $u_{\tau}=28.3 \mathrm{~m} / \mathrm{s}$ and a friction Mach number of $M_{\tau}=u_{\tau} / \sqrt{\gamma R T_{w}}=0.091$. It can be assumed that the incoming boundary layer at $x_{8}$ is turbulent since the viscous linear region and the logarithmic overlap region are clearly distinguishable with the sonic line residing in the inner region of the boundary layer. It is important to point out that an attempt was made to correct for wall curvature (Fernholz and Warnack, 1998), compressibility effects (van Driest's effective velocity) and pressure gradient (Karman-type closure that relates Cole's wake parameter to Clauser's equilibrium pressure gradient parameter using the polynomial expression of Das (1987) for non-equilibrium flows) in order to better correlate our findings with those of the ZPG flat plate studies. The effort proved unsuccessful given the strong changing pressure gradient that forms in these kinds of flows.

The local free-stream velocity, Mach number and boundary layer thickness are assessed from the RANS model and used to compute a SWBLI characteristic frequency. The values are tabulated in table 1 at four axial locations $\left(x_{3}-x_{5}, x_{8}\right)$ and will be relevant to subsequent analyses in $\S 3$ and $\S 4$. The Reynolds number based on $\delta$ (defined as $R e_{\delta}=u_{e} \delta / \nu_{e}$ ), is also provided. As for the Reynolds number based on friction velocity, 

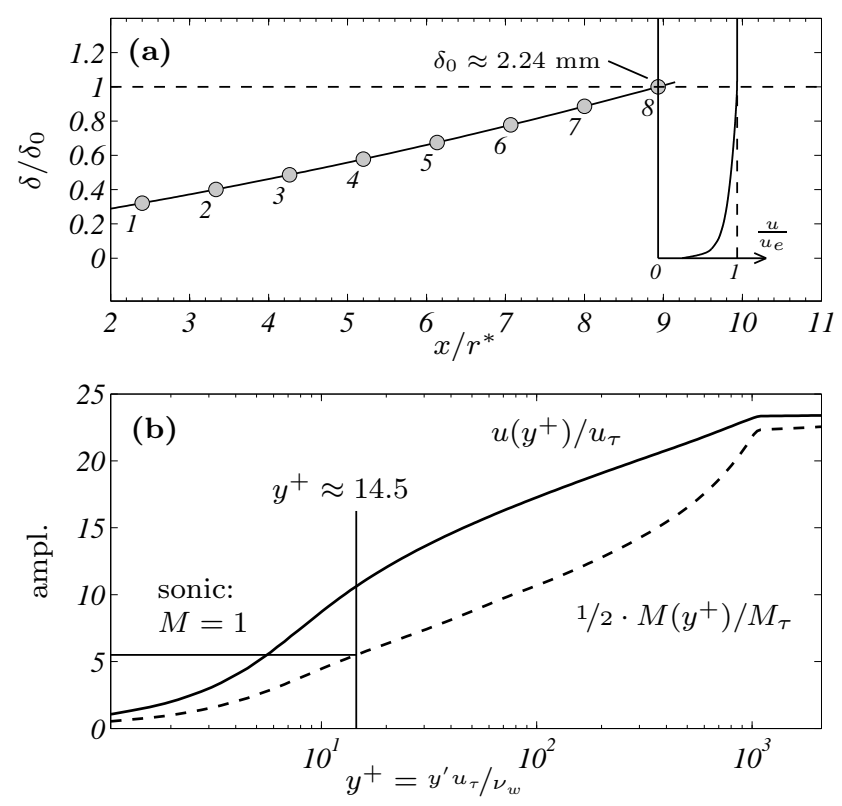

Fig. 6 a Boundary layer thickness $\delta / \delta_{0}$ as function of axial distance where $\delta_{0}$ is the thickness at location $x_{8}$ and is valued at $\approx 2.24 \mathrm{~mm}$. b Boundary layer profiles of velocity $\left(u^{+}\right)$and Mach number $\left(M^{+}\right)$using inner scales with $u_{\tau}=28.3 \mathrm{~m} / \mathrm{s}$ and $M_{\tau}=0.091$

Table 1 Outer scaling variables of the boundary layer obtained from the RANS simulation visualized in Fig. 5

\begin{tabular}{cccccc}
\hline loc. & $M_{e}$ & $\begin{array}{c}u_{e} \\
{[\mathrm{~m} / \mathrm{s}]}\end{array}$ & $\begin{array}{c}\delta \\
{[\mathrm{mm}]}\end{array}$ & $\begin{array}{c}u_{e} / \delta \\
{[\mathrm{kHz}]}\end{array}$ & $R e_{\delta}$ \\
\hline$x_{3}$ & 3.39 & 631 & 1.09 & 580 & $7.5 \cdot 10^{4}$ \\
$x_{4}$ & 3.54 & 639 & 1.29 & 494 & $7.5 \cdot 10^{4}$ \\
$x_{5}$ & 3.68 & 645 & 1.50 & 431 & $8.1 \cdot 10^{4}$ \\
$x_{8}$ & 4.07 & 662 & 2.24 & 295 & $8.5 \cdot 10^{4}$ \\
\hline
\end{tabular}

this is computed using the $\delta_{0}$ at $x_{8}$ and is found to be $R e_{\tau}=\delta_{0} u_{\tau} / \nu_{w}=1050$. Given the roughness of the nozzle's interior surface (around $100 \mu \mathrm{m}$ ), the boundary layer in the supersonic part of the nozzle is believed to have transitioned naturally early on and to have developed under near-adiabatic wall temperature conditions.

A schematic of the stationary SWBLI of the incipient separation shock, whose intermittent region resides around location $x_{8}$, is shown in Fig. 5b. The incoming boundary layer thickness at this location is $\delta_{0} \approx$ $2.24 \mathrm{~mm}$, the local Mach number is $M_{e}\left(x_{8}\right)=4.1$, the local velocity is $u_{e}\left(x_{8}\right)=662 \mathrm{~m} / \mathrm{s}$, and the annular separation shock is inclined at an angle of about $\beta=28^{\circ}$ with respect to the wall. The latter is measured from density and temperature contours extracted from the RANS model since the separation shock expresses itself as a sharp discontinuity within these contours. Given these parameters, it is estimated that our dynamic wall
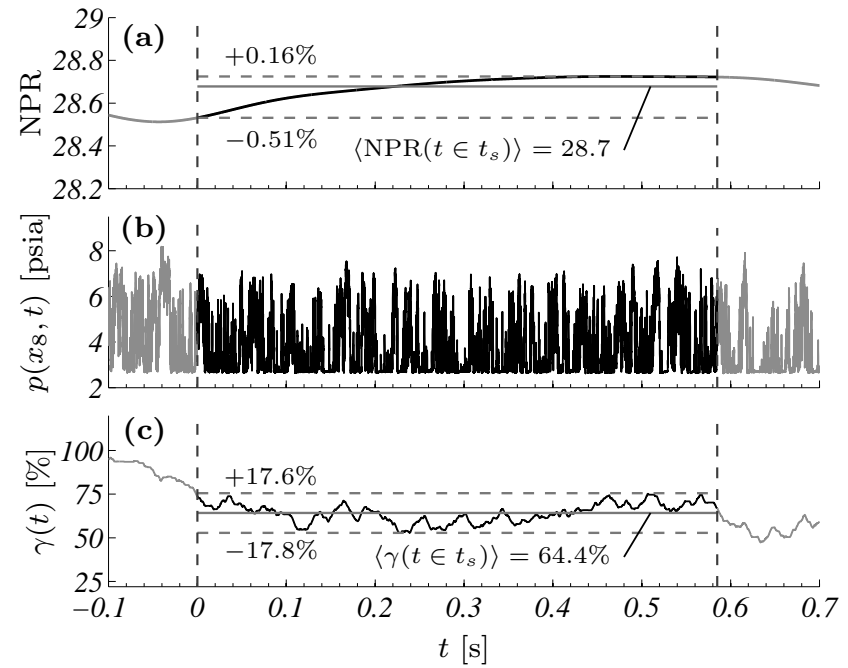

Fig. 7 a NPR trend during fixed NPR operation; the mean and maximum deviation bounds are indicated. $\mathbf{b}$ pressure trace at location $x_{8}$; the signal is adjusted to account for small drift in NPR. c window-averaged intermittency of the pressure time-series at location $x_{8}$; window size of $75 \mathrm{~ms}$

pressure measurements at location $x_{8}$ are resolving fluctuations up to a non-dimensional frequency of $\tilde{f}_{r}=$ $f_{s} / 2 \cdot \delta_{0} / u_{e}=0.068$. For ZPG boundary layer flows, Ganapathisubramani et al (2009) has shown how the large-scale unsteadiness associated with low-frequency shock motion is of the order of $f \delta_{0} / u_{e} \approx 0.008-0.025$. While the current study encompasses an incoming boundary layer with a strong favorable pressure gradient (FPG) and has wall curvature with surface roughness, the findings from the ZPG boundary layer flows should provide some reasonable guidance. And so, while it is currently not possible to accurately resolve the full spectrum of scales at this location $\left(\tilde{f}_{r} \gg 1\right)$ due to transducer size, transducer sensitivity and the upper limit of the resolved frequencies (severe spatial and temporal filtering, see Smits and Dussauge, 2006), the large-scale unsteadiness can still be studied.

\section{Stationary SWBLI}

Here we focus on the unsteady behavior of the shock foot, as well as conditional features downstream for the case of stationary operation of the nozzle.

\subsection{Flow details}

A more detailed inspection of Fig. 4a is shown in Fig. 7 to explain how the data was acquired and subsequently analyzed. Only a portion of the experimental run with a fixed NPR operating condition was selected. 


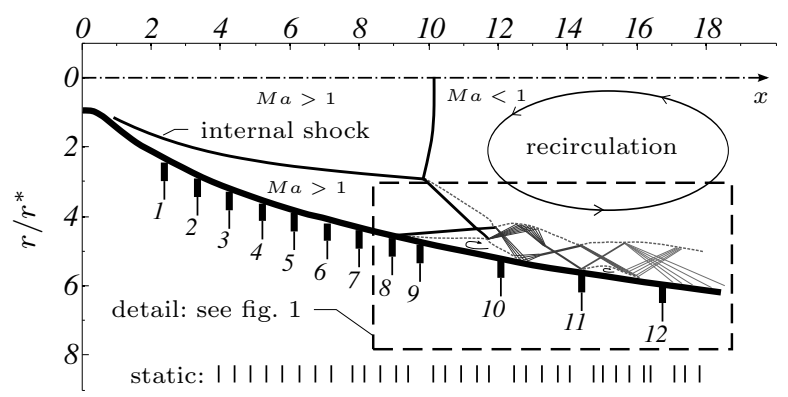

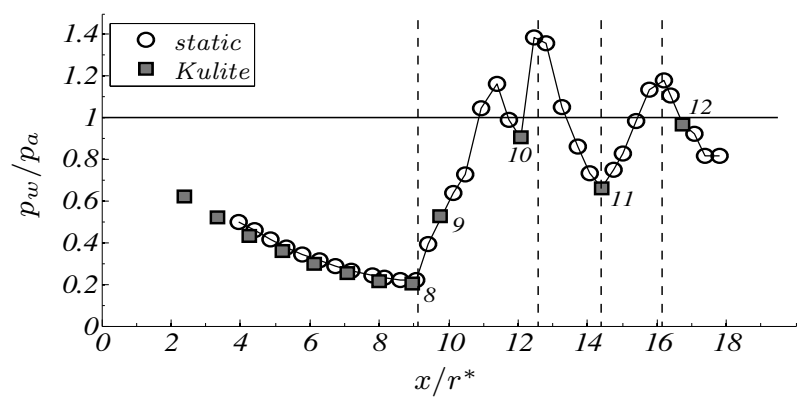

Fig. 8 Illustration of the internal shock structure during RSS state in a TOP nozzle. Locations of the Kulites (numbered) and static pressure ports (vertical lines) are indicated and the associated static wall pressure profile is shown for the fixed NPR experiment

Based on a threshold procedure described in $\S 3.2$, the intermittency (fraction of time that the shock foot is upstream of the location of observation; location $x_{8}$ in this case) over a $75 \mathrm{~ms}$ of data is constructed and plotted as the moving-window-averaged intermittency in Fig. 7c. It can be seen that the window-averaged intermittency, for $t \in[0,0.58] \mathrm{s}$, fluctuated around the mean with a maximum deviation of about $18 \%$. And so, only this portion of the stationary data is used throughout this section. The resultant time-averaged NPR is $\langle\mathrm{NPR}\rangle=28.7$, with deviation bounds close to $1 \%$.

A comparison of the static wall pressure profile and the mean absolute pressure obtained from the dynamic transducers is shown in Fig. 8 (bottom). The key features of the shock wave pattern inferred from this wall pressure profile are also shown (top) which depicts a classical RSS structure comprising two trapped annular separation bubbles. The locations of the dynamic pressure transducers are shown (numbered 1 to 8 for $x_{1}-x_{8}$ ) with $x_{8}$ residing in the intermittent region of the incipient separation shock. Transducers $x_{9}$ and $x_{10}$ are located downstream in the separated region (indicated by two dashed vertical lines) while $x_{11}$ is near the impingement of the incident shock (see Fig. 1).

Scalar statistics from the dynamic wall pressures are shown in Fig. 9 and comprise (from top to bottom) a dimensionless standard deviation (normalized by the wall pressure: $\left.\sigma_{p} / p_{w}\right)$, skewness of the pressure $S(p)$, and the Wave Steepening Factor (WSF). The latter is defined

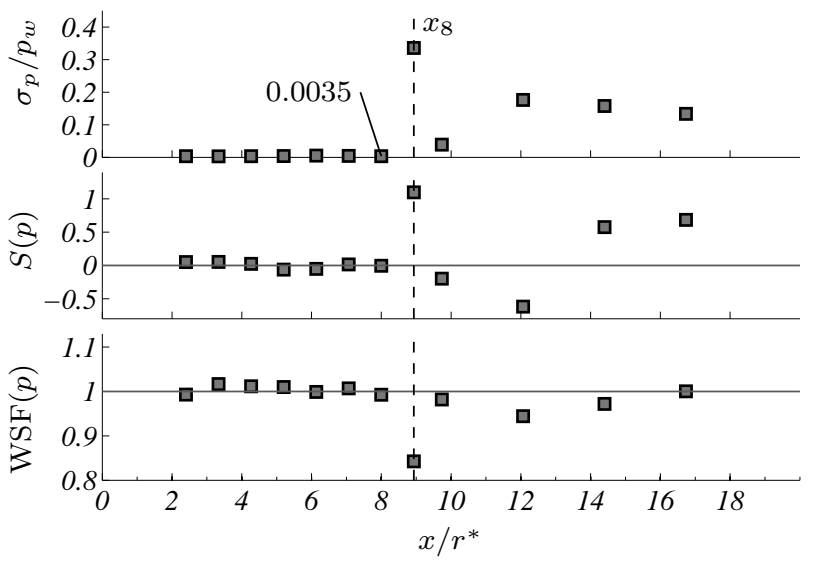

Fig. 9 Scalar statistics of the dynamic wall pressures. From top to bottom: normalized standard deviation, skewness and the Wave Steepening Factor (WSF)

as the modulus of the average negative slope divided by the average positive slope in the pressure recording (Gallagher and McLaughlin, 1981). The range of WSF is thus bounded by 0 and 1 , where 1 corresponds to a harmonic wave (symmetric) and 0 to a sawtooth wave. As a consequence of $x_{8}$ being located in the intermittent region of the separation shock, $p\left(x_{8}\right)$ registers unsteady shock sweeps. Although the skewness is high during such events, as is expected, the WSF is relatively close to one: $\left.\operatorname{WSF}(p)\right|_{x_{8}}=0.85$. This suggests that, on average, the shock is traveling slightly faster upstream than it is downstream as a value of zero or infinity would correspond to gross differences between upstream and downstream speeds of the shock motion over the transducer.

Shock motion unsteadiness appearing at location $x_{8}$, and how that correlates with the downstream wall pressure, is now studied by conditionally averaging the pressure time series. Sample pressure time-series at $x_{7}$ - $x_{12}$ are first shown in Fig. 10, alongside their Probability Density Functions (PDFs) relative to a Gaussian (dashed line). It is well-known that large-scale fluctuations and fine-scale turbulence dominate the wall pressure footprint downstream and upstream of the shock foot, respectively ( the raw time series. As was apparent from $\S 3.1$, the signal at $x_{8}$ is a manifestation of the wall pressure signatures that are being sensed both upstream and downstream of the separation shock foot. At instances when the foot is downstream of $x_{8}$, the resolved range of unsteady pressure encompasses relatively small fluctuations whose mean pressure reflects that of an attached, supersonic, boundary layer. Conversely, when the shock foot is upstream, pressure recordings show large fluctuations that are characteristic of a separated flow. By 

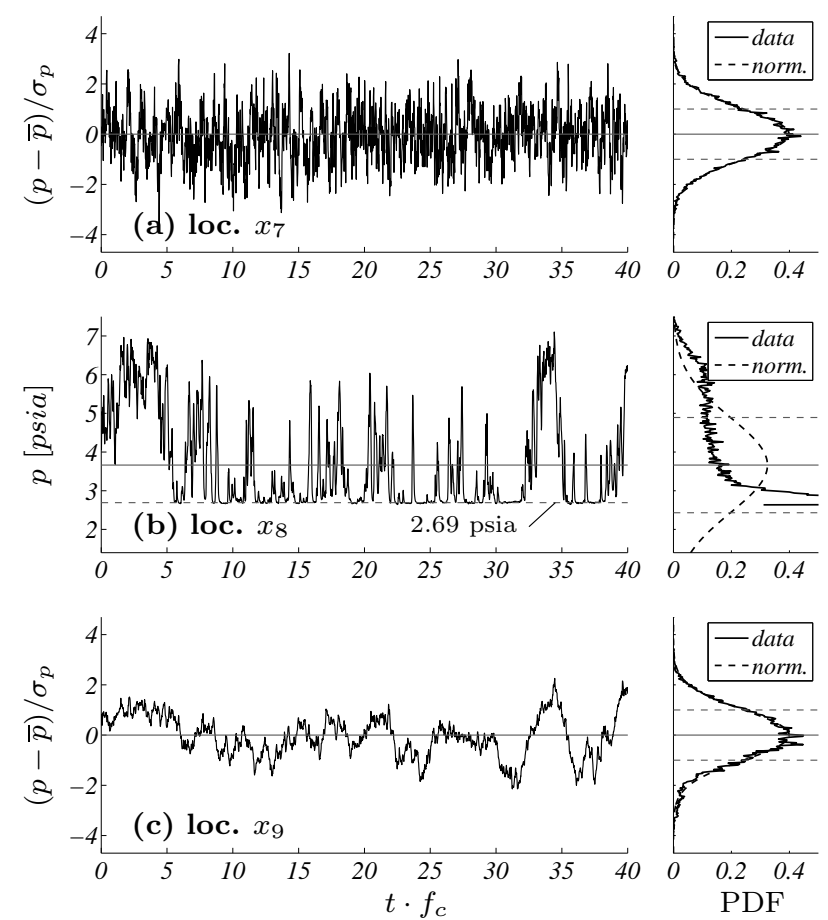
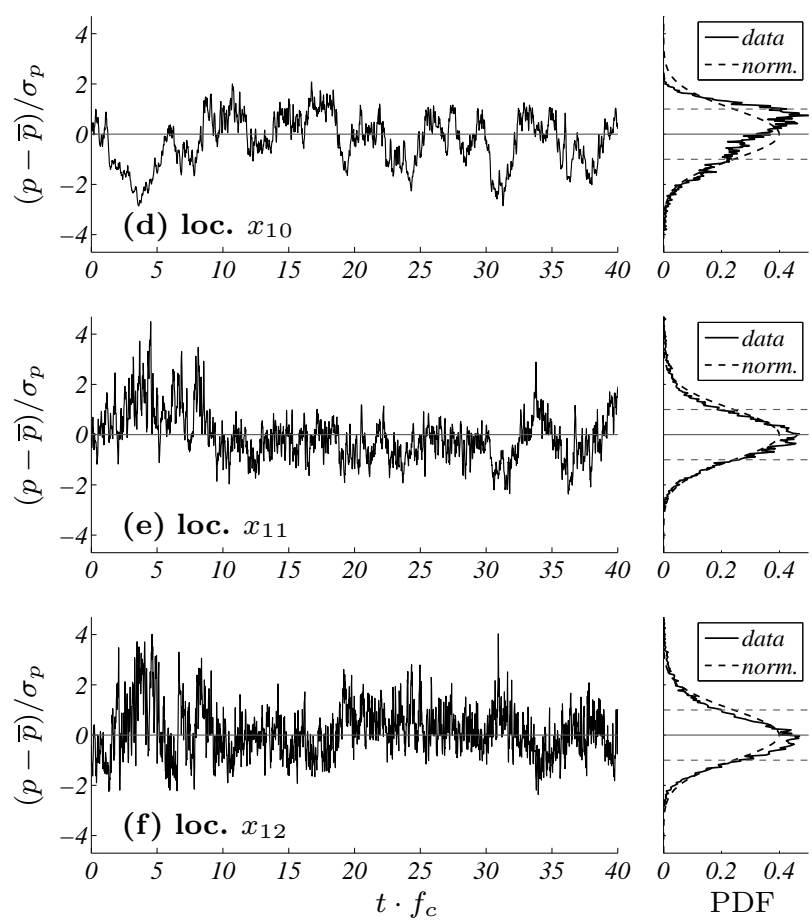

Fig. 10 Time-synchronized wall pressure time series at location $x_{7}-x_{12}$ with associated PDFs generated from all stationary data: $t \in[0,0.58] \mathrm{s}$. For reference, $t \cdot f_{c}=40$ equals $t=58.3 \mathrm{~ms}$ or $t u_{e} / \delta_{0}=1.71 \cdot 10^{4}$ at location $x_{8}$. Signals were high-pass filtered at $5 \mathrm{~Hz}$ and the pressure time series at $x_{8}$ (conditional point) is adjusted to account for small drifts in NPR

visual inspection, one could see a high degree of correlation between pressure time series at $x_{8}$ and subsequent downstream locations $\left(x_{9}-x_{11}\right)$. Similar observations in the pressure recordings in the intermittent regions of SWBLIs associated with canonical compression ramps can be found in the literature (Dolling and Or, 1985; Dolling and Brusniak, 1989; Erengil and Dolling, 1991).

\subsection{Variable-window conditional averaging}

An assessment of the relationship between shock motion and wall pressure unsteadiness is now examined by exploiting a novel approach described by Dolling and Brusniak (1989). By determining instances in time when the shock foot moves over a transducer residing in the intermittent region $\left(x_{8}\right)$, one can isolate signatures corresponding to either upstream or downstream motions of the shock foot; here we will choose to use $x_{8}$ as the conditional point. This approach is summarized in Fig. 11, which duplicates Fig. 3 of Erengil and Dolling (1991), and works as follows.

1. When the shock foot is downstream of $x_{8}$, the pressure recording represents a locally undisturbed and attached turbulent boundary layer. By manually record ing the mean pressure at these time instances, a socalled pressure floor, at location $x_{8}$ and denoted as $\bar{p}_{f}$, is established.
2. Next, two thresholds are defined: $T_{1}=\bar{p}_{f}+3 \sigma_{p}\left(x_{7}\right)$ and $T_{2}=\bar{p}_{f}+6 \sigma_{p}\left(x_{7}\right)$, so that the pressure timeseries $p\left(x_{8}\right)$ can be decomposed into regions where the separation shock foot is either upstream (boxcar function is on) or downstream of $x_{8}$ (boxcar function is off). That is, the boxcar function is switched on when $p\left(x_{8}\right)>T_{2}$ and switched off when $p\left(x_{8}\right)<T_{1}$. Thus, when a 'rise' of the boxcar function appears, the shock moves in the upstream direction over $x_{8}$, and adversely, when a 'fall' is identified, the shock foot moves downstream. Note that these shock passages, either upstream or downstream, are the only pertinent time-scales of the shock motion unsteadiness that we consider in this work.

3. The shock zero-crossing frequency corresponding to the average rise- or fall-times in the pressure waveform can then be computed as

$f_{c}=\frac{1}{\frac{1}{N} \sum_{i=1}^{N} T_{i}}=\frac{1}{\left\langle T_{i}\right\rangle}=686.1 \mathrm{~Hz}$

with an intermittency factor $\gamma$, defined as the fraction of time that the shockfoot is upstream of $x_{8}$, determined to be $\gamma=64.4 \%$ (also identified in Fig. 7c).

In addition to these steps, one may choose to use a -wavelet-based (or Fourier-based) filter to separate the shock motion component of the signal from the finescale turbulence prior to conditional averaging (see Poggie and Smits (1997)). Based on earlier studies by Thomas 

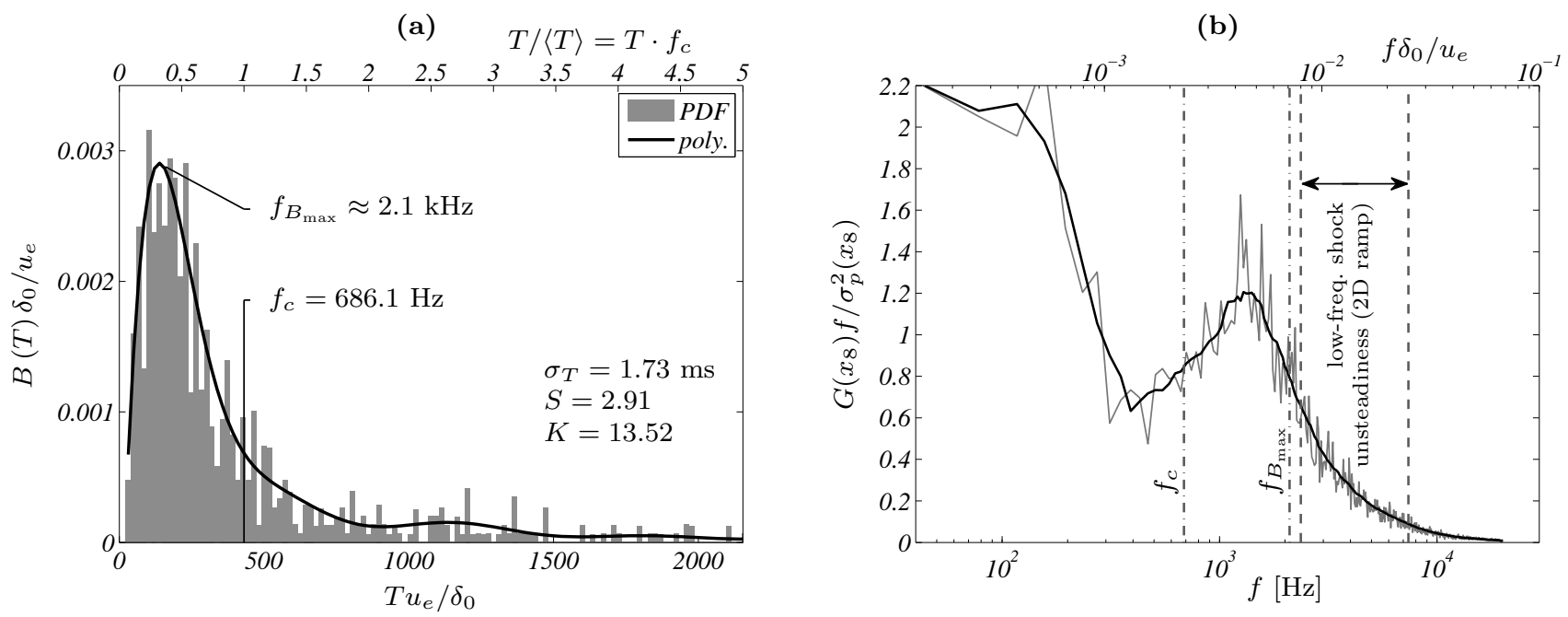

Fig. 12 a Average PDF of the rise- and fall-intermittence created out of 396 shock passages. Note that the distribution does not fall in the catergory of a $\chi^{2}$, Rayleigh or $\Gamma$ distribution. $\mathbf{b}$ PSD of pressure time-series at location $x_{8}, \sigma_{p}^{2}\left(x_{8}\right)=1.514$ psia ${ }^{2}$

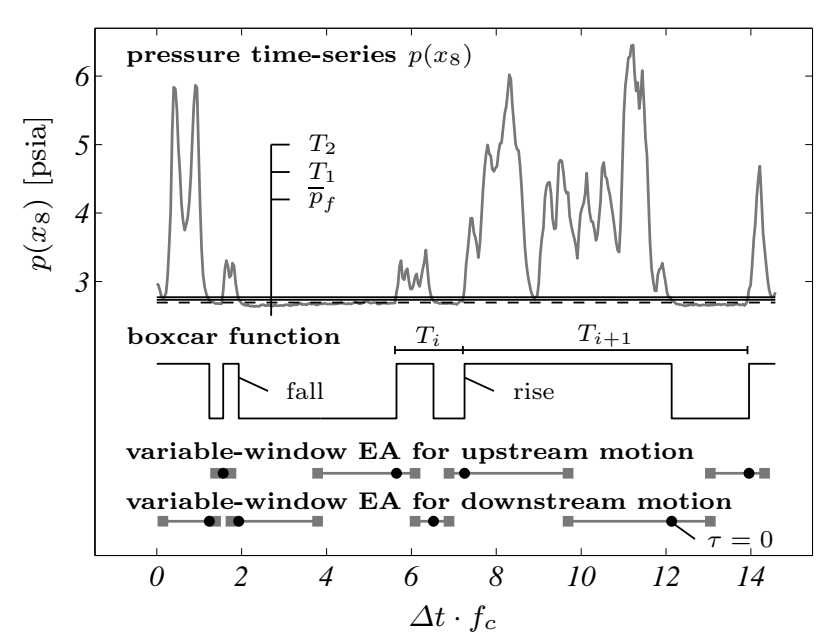

Fig. 11 Illustration of the boxcar conversion technique and the variable-window ensemble averaging, following Erengil and Dolling (1991). Thresholds are taken as: $T_{1}=\bar{p}_{f}+$ $3 \sigma_{p}\left(x_{7}\right)$ and $T_{2}=\bar{p}_{f}+6 \sigma_{p}\left(x_{7}\right)$

et al (1994) as well as simple observations, reveal that these shock passages are non-periodic in nature, and hence, the shock zero-frequency $f_{c}$ has limited physical relevance to the shock motion unsteadiness. Therefore, an average PDF of the rise- and fall-intermittence (time between successive shock crossings) is overlaid a higher order polynomial approximation in Fig. 12a. The mean of this PDF is equal to the shock zero-crossing frequency, which, in normalized form, is equal to $f \delta_{0} / u_{e} \approx$ $0.0023^{1}$. The most probable frequency at which the shock crosses $x_{8}$ is equal to $f \delta_{0} / u_{e} \approx 0.0073$ (peak of the $\mathrm{PDF}$ at $2.1 \mathrm{kHz}$ ). And so, a plausible shock cross-

1 Note that the shock zero-crossing frequency is not equal to the mean frequency of shock oscillations. ing frequency is approximately three times the shock zero-crossing frequency. One can see that the PDF in Fig. $12 \mathrm{a}$ is highly skewed about the mean (skewness of $S=2.91$ and kurtosis of $K=13.52$ ); similar features have also been observed in Mach 1.5 compression ramp experiments (Thomas et al, 1994). The energy spectrum of the pressure time-series at $x_{8}$ is shown in Fig. $12 \mathrm{~b}$ with two humps being manifest. The first resides at frequencies below $300 \mathrm{~Hz}\left(f \delta_{0} / u_{e}<0.001\right)$, while the second comprises a peak centred around $1,300 \mathrm{~Hz}\left(f \delta_{0} / u_{e} \approx\right.$ 0.004 ). When we translate the first energetic peak (with a frequency of $150 \mathrm{~Hz}$ ) to a time-scale, we obtain 1/150= $0.0067 \mathrm{~s}$, or in non-dimensional form as $\left(t \cdot f_{c}\right)=4.6$. This value agrees well with a direct inspection of the time-series in Fig. 10b when looking at possible zero crossings. Superposed on the low-frequency shock motion is the higher-frequency, lower-amplitude, content of $1,300 \mathrm{~Hz}$ and this is likely caused by boundary layer pressure fluctuations, which differs from the frequency associated with the axial fore and aft movement of the shock. The discrepancies between the humps in Fig. $12 \mathrm{~b}$ and the low-frequency shock motion unsteadiness identified during the $2 \mathrm{D}$ compression ramp study of Ganapathisubramani et al (2009) $\left(f \delta_{0} / u_{e} \approx 0.008-\right.$ $0.025)$ are attributed to the FPG of the incoming boundary layer, relative to the ZPG study of Ganapathisubramani et al (2009), and the additional surface roughness.

\subsubsection{Pressure time histories}

Following the procedure outlined by Erengil and Dolling (1991) and illustrated in Fig. 11, conditional time histories of the fluctuating wall pressure at locations $x_{8}-x_{12}$ are shown in Fig. 13. As the number of samples between 

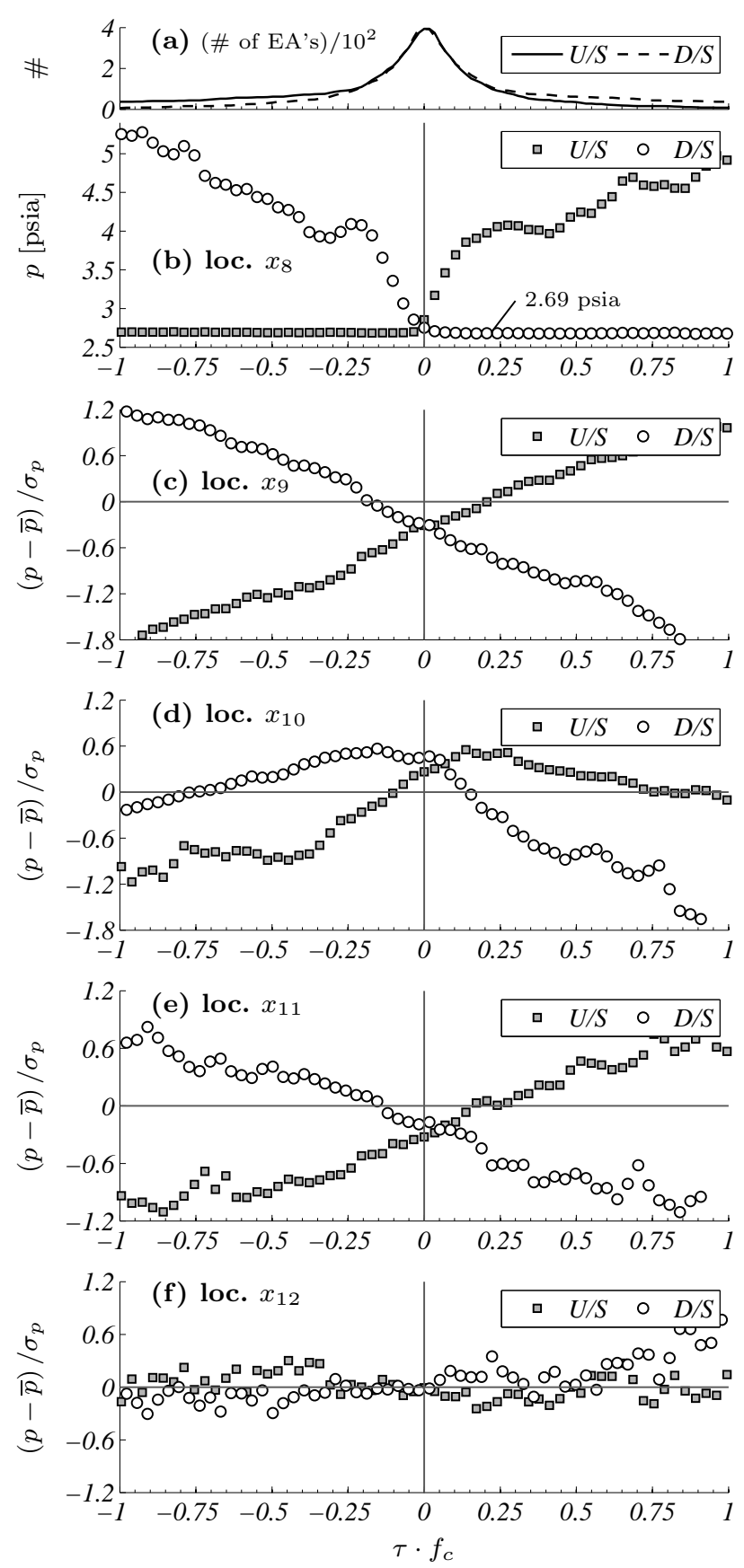

Fig. 13 Ensemble-averaged pressure histories at location $x_{8}$ - $x_{12}$ (a-f) for upstream (U/S) and downstream (D/S) shock motions; $x_{8}$ is the conditional point. For clarity, temporal points are shown with half the available resolution

successive upstream or downstream motions increases, the number of ensembles that one can average over decreases, as demonstrated in Fig. 13a. This quantitative analysis shows how motions of the incipient separation shock and pressure fluctuations in the separated region downstream $\left(x_{9}-x_{10}\right)$ are out of phase; the pressure decreases when the shock moves downstream and vice versa. This result is indicative of a long intermittent region (in terms of $\delta$ ), since the out-of-phase behaviour can be explained by translating the static wall pressure profile along with the shock motion. A similar hypothesis by Plotkin (1975) was used to develop a simple model for the shock wave motion. Interestingly, the results contradict the behaviors seen in bounded separation bubbles that form just downstream of the shock foot in front of canonical compression ramps (Erengil and Dolling, 1991) where shock motion and pressure experience an in-phase relationship. Additional measurements are required to determine if our observations are indeed the result of more extensive motions of the shock foot (i.e. the shock foot is not anchored to a geometric feature inside the smooth expanding nozzle contour, as opposed to a compression ramp or blunt fin in the case of classical SWBLI studies reported in the literature). A densely spaced stream wise array of pressure transducers in the intermittent region would reveal this behaviour in more detail, but ideally, internal flow measurements would provide much more meaningful results; internal flow measurements are currently unavailable at this time.

As for the transducers located at $x_{9}$ (Fig. 13c) and $x_{11}$ (Fig. 13e) the ensemble averaged pressure time series at these locations manifest a high degree of similarity. Location $x_{11}$ seems to be just downstream of the stream wise point where the incident shock impinges on the wall and re-separates the flow. The pressure timeseries $p\left(x_{11}\right)$, Fig. 10e, does not provide any indication that the incident shock is moving downstream of $x_{11}$, as that would result in a low-amplitude signal resembling an attached supersonic boundary layer. Therefore, the pressure time histories at $x_{11}$ show that the pressure fluctuations in the second separation bubble are inphase with pressure fluctuations in the first separation bubble, and hence out-of-phase with the motion of the incipient separation shock foot. This implies that the shock system drawn in Figs. 1 and 8, moves unsteadily in a rigid fashion and at relatively low frequencies. This is insightful to understanding the increased loads that form during the end-effects regime (Nave and Coffey, 1973; Nguyen et al, 2003) and why there are no other large amplitude unsteady loads when the other subsonic annular separation bubbles exit the nozzle. Finally, an absence of correlation between the attached supersonic flow at location $x_{12}$ and the motion of the separation shock is gleaned from the pressure time history in Fig. 13f. This is not surprising given the low levels of energy in the pressure fluctuations of the boundary layer, as well as the deterioration of the correlation by the two upstream shocks, regions of separated flow, and flow reattachment. Finally, the intersections of the 
$\mathrm{U} / \mathrm{S}$ and $\mathrm{D} / \mathrm{S}$ curves in Figs. 13c, $\mathrm{d}$ and e appear at a small positive delay $(\tau>0)$ which is indicative of a finite propagation time for the change at the conditional point to reach the subsequent stations $x_{9}$ to $x_{11}$ and/or an asymmetric behavior (hysteresis) in the U/S and $\mathrm{D} / \mathrm{S}$ motions of the signatures at $x_{9}$ to $x_{11}$.

\section{Non-stationary SWBLI}

Having now established an understanding for the lowfrequency shock motion unsteadiness during stationary operations, we turn our attention to transient start-ups and shut-downs. We begin this section ( $\S 4.2)$ by reviewing some of the key flow features that appear during transients, followed by an overview of time-frequency analysis in $\S 4.2$. In $\S 4.3$, we focus our attention on transient sweeps of the shock foot -or SWBLI- over location $x_{8}$ in order to compare the behavior of the shock foot unsteadiness during transient operations to those that form during the non-transient operations discussed in $\S 3.2$. In closing, the effect that the transient start-up rate has on the low-frequency unsteadiness exerted on the global system is then described in $\S 4.4$.

\subsection{Temporal inspection}

By operating the nozzle in a transient manner, the shock system varies in strength and structure as it sweeps along the nozzle wall. Sample time-series of all twelve dynamic transducers at location $x_{1}$ to $x_{12}$ are presented in Fig. 14b for the second ramping case shown in Fig. 4b. Initially, the internal flow exhibits a FSS state whereby a single separation shock sweeps over locations $x_{1}$ to $x_{5}$ in a staggered sense. A sweeping of the intermittent region over locations $x_{3}, x_{4}$ and $x_{5}$ are highlighted by windows A, B, and C, respectively, and will be used for subsequent analyses in $\S 4.2$. While operating in a FSS state, all downstream transducers record pressure fluctuations associated with the subsonically entrained flow. At a NPR of 24.3, the flow state transitions from FSS to RSS whereby the subsonic entrained flow gives way to intense pressure fluctuations associated with expansion and compression waves that bound separation bubbles. As the shock pattern pushes out of the nozzle, intense wall pressure fluctuations form at locations $x_{11}$ and $x_{12}$ and is caused by the intermittent relief of pressure that occurs when the first bubble opens up to atmosphere; this was mentioned earlier and is referred to as the 'end-effects regime' (Nave and Coffey, 1973; Nguyen et al, 2003). For this particular ramp, the incipient separation shock foot resides between $x_{10}$ and $x_{11}$ when the maximum NPR of approximately 60 is (a)

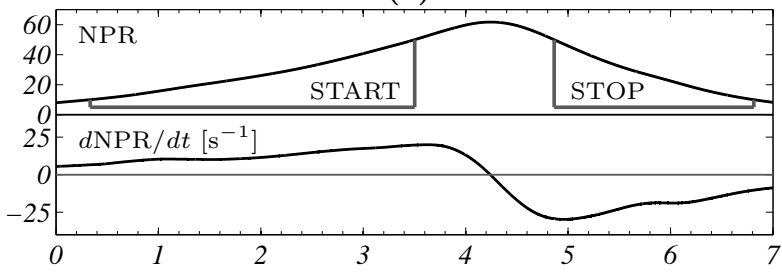

(b)

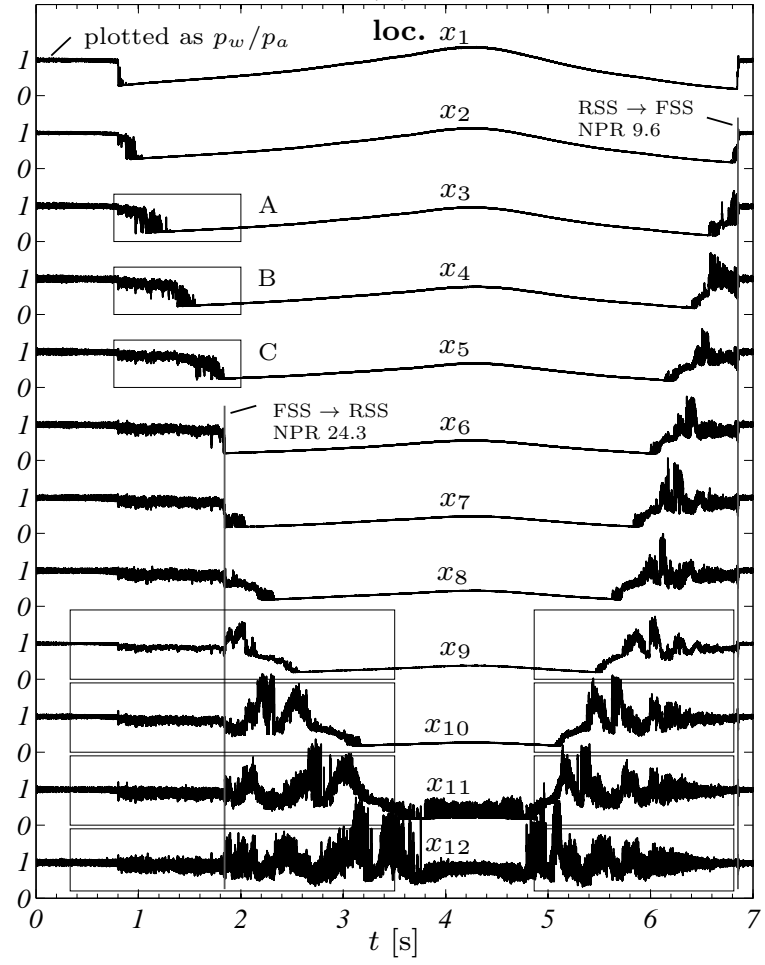

Fig. 14 a NPR trend of ramp 2. b Pressure time-series at location $x_{1}$ to $x_{12}$ during start-up and shut-down of ramp 2

reached at $t=4.2 \mathrm{~s}$. During shut-down, a hysteresis phenomenon occurs whereby the RSS to FSS transition appears around NPR $\approx 9.6$ (Frey and Hagemann, 2000; Ruf et al, 2009; Wang, 2009).

\subsection{Time-frequency analysis}

A time-frequency analyses (Cohen, 1989; Farge, 1992) of the fluctuating wall pressure is performed in a similar fashion as Baars and Tinney (2013) in order to view the spectral properties of the wall pressure data as a function of time. The process works by convolving -in the frequency domain- a mother wavelet $\psi$ with the fluctuating wall pressure $p\left(x_{j}, t\right)$ at location $x_{j}$ thereby producing complex-valued wavelet coefficients according to

$\tilde{p}\left(x_{j} ; l, t\right)=\int p\left(x_{j}, t^{\prime}\right) \psi^{*}\left(\frac{t^{\prime}-t}{l}\right) d t$, 
where $l$ is a time-scale of the predefined wavelet. Each wavelet time-scale can be transformed to an equivalent Fourier frequency $f$, and so, the fluctuating wall pressure is decomposed in time-frequency space. For the purposes of this study, we are interested in the energy density, known as the Wavelet Power Spectrum (WPS), which is taken as

$E(x ; l, t)=\frac{|\tilde{p}(x ; l, t)|^{2}}{l}$,

and is then converted to Fourier frequency to obtain the double-sided WPS, denoted as $E(x ; f, t)$. The current application comprises a resolved band of frequencies ranging from $10 \mathrm{~Hz}$ to $f_{s} / 2$, spaced uniformly in a logarithmic frame using 81 frequency scales. The Mexican hat wavelet is employed as the mother wavelet and is the $2^{\text {nd }}$ derivative of a Gaussian, defined as

$\psi(t / l)=\left(1-|t / l|^{2}\right) e^{-|t / l|^{2} / 2}$.

Implications of the wavelet choice on the time- and frequency-resolution of the WPS is discussed extensively in the literature (Farge, 1992; Addison, 2002). For transients that encompass small time-scales, the timeaveraged double sided wavelet spectra can be resourceful and is calculated using $\bar{E}=1 / T \int_{T} E d t$, where $T$ is the time-domain over which the averaging is performed. As for stationary signals, the single-sided global wavelet spectrum (time-average spectrum of the complete signal) compares well with a filtered Fourier spectrum: $G\left(x_{j} ; f\right)=2 \cdot \bar{E}\left(x_{j} ; f\right)$.

We now turn our attention to the energy content in the pressure time-series at locations $x_{3}, x_{4}$ and $x_{5}$ during sweep-over of the incipient separation shock (FSS state). The pressure time-series were shown in Fig. 14b with three domains of interest, highlighted by windows $\mathrm{A}, \mathrm{B}$ and $\mathrm{C}$, comprising sweep-overs of the intermittent region. The Mexican hat $\mathrm{WPS}^{2}$ are shown directly above the raw pressure time series in Fig. 15. It is important to mention that no data is accessible at these locations when the nozzle is operated at a fixed NPR at which the intermittent region of the shock foot is centred around one of these locations; in $\S 4.3$, the sweep of the incipient separation shock foot over location $x_{8}$ is considered, for which a stationary counterpart is available for comparison. Nonetheless, it is evident that the energy resides within two frequency bands during these sweeps. The first coincides with a sweeping of the intermittent region and resides at very low frequencies $(10-50 \mathrm{~Hz})$. This is a consequence of gross changes to the mean wall pressure during the shock sweep, which

2 Overlapping signal partitions of $N=2^{14}$ samples are transformed. The regions inside the cone of influence (Farge, 1992), for $10 \mathrm{~Hz}<f<f_{s} / 2$, are presented in a continuous fashion throughout the article. is most significant in Figs. 15a, b and c around 1.2, 1.4 and 1.7 seconds, respectively. The duration of these sweeps comprises time scales on the order of $0.1-0.4 \mathrm{~s}$ which correlates well with the increased low-frequencies that the wavelet spectra manifest. And so, this low frequency unsteadiness is due to changes in the mean wall pressure, as opposed to shock intermittence. The second high-energy band resides around $400 \mathrm{~Hz}$, or a dimensionless frequency of $f \delta_{0} / u_{e} \approx 0.001$. Changes in the boundary layer parameters are too subtle during these snippets of time and so the ordinate scale is made dimensionless by assuming the values in Table 1 to be constant. The mechanism underlying this $400 \mathrm{~Hz}$ energy band is hypothesized to be transonic resonance (Zaman et al, 2002), which can form between the separation shock and the exit plane of a highly overexpanded nozzle. The shock system compares to a vibrating piston that locks-in with the downstream recirculating flow and is analogous to a standing wave that forms in an open-ended pipe. The fundamental acoustic resonance forms from one-quarter of the standing wave pattern or any of its odd harmonics. During the FSS state, the downstream flow of the incipient separation shock and Mach disk has become pressure balanced with the ambient and the sound speed of the gas is therefore assumed to be $a_{\infty}=343 \mathrm{~m} / \mathrm{s}$. The distance between the incipient separation shock, or Mach disk in the center of the nozzle, and the nozzle lip is approximately $L=0.23 \mathrm{~m}$. By assuming that a $1 / 4$-wavelength resides in this region, the resonance tone would be $a_{\infty} /(4 \cdot L)=373 \mathrm{~Hz}$. This frequency is comparable to the $400 \mathrm{~Hz}$ energy band. Furthermore, Baars et al (2012) showed spectra of the wall pressure downstream of the FSS incipient separation point during stationary operation at a NPR of approximately 25. The spectra exhibited strong tones at a similar frequency of $400 \mathrm{~Hz}$.

\subsection{Transient sweeps of SWBLIs over location $x_{8}$}

In an effort to isolate the effects that large transient flow motions have on SWBLI, the pressure footprint registered at $x_{8}$ is analysed during a sweeping of the intermittent region associated with the incipient separation shock. It is clear from Fig. 14 that the internal nozzle flow transitions to a RSS state before the incipient shock foot reaches $x_{8}$. And so, it is important to realize that we only focus on very localized unsteady features at the nozzle wall (location $x_{8}$ during the sweep of one shock foot) while the nozzle wall is concurrently exposed to a tremendous amount of unsteadiness exerted by the entire RSS shock train. Furthermore, while Fig. 14 only presents the time-series corresponding to ramp 2, it is in fact the case that for all other ramps, 

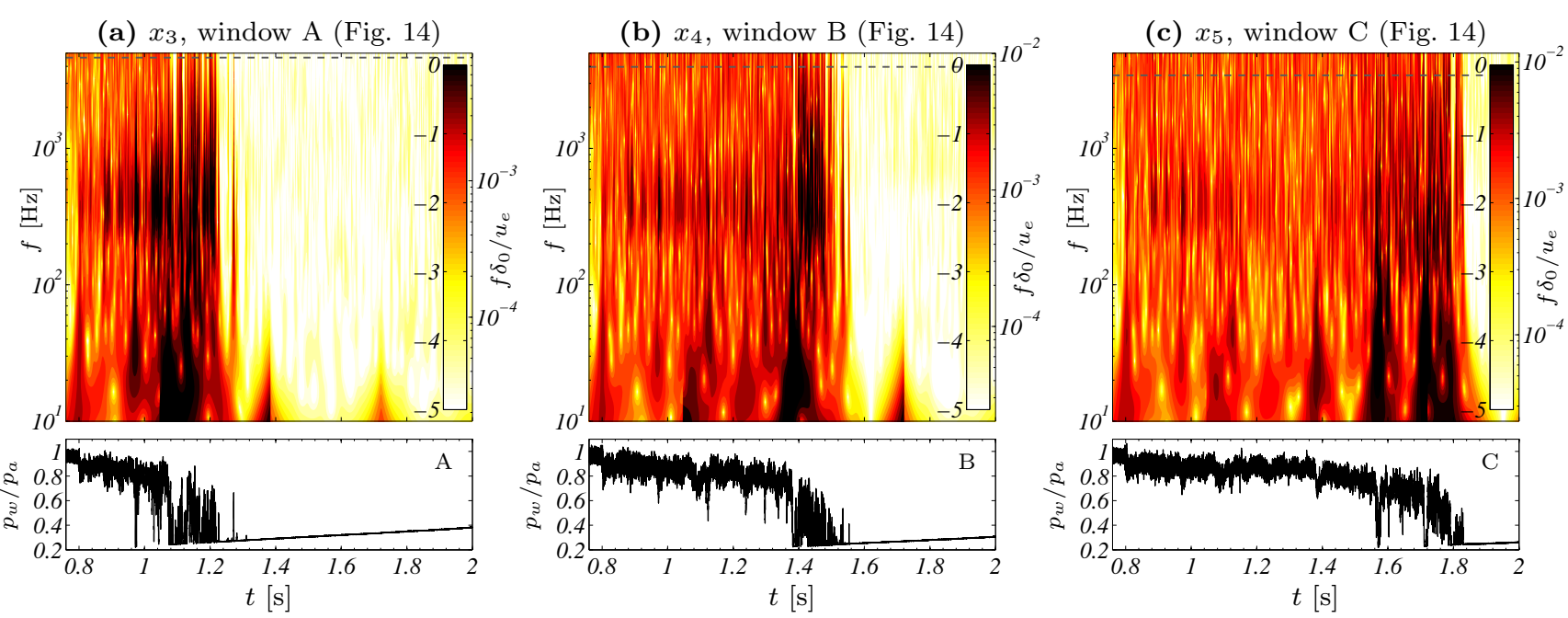

Fig. 15 Pre-multiplied Mexican hat WPS, contour is $\log _{10}\left(E\left(x_{j} ; f, t\right) \cdot f\right)$ in $\left[\mathrm{psia}^{2}\right]$, for the pressure time-series that include the FSS shock foot sweep at location $\mathbf{a} x_{3}, \mathbf{b} x_{4}$ and $\mathbf{c} x_{5}$. The raw time signal is synchronously shown. Note that time is not normalized. Frequency is shown in normalized form on the right ordinate; $u_{e} / \delta_{0}$, and values for frequency normalization are indicated in table 1. The lower threshold for stationary low-frequency shock motion unsteadiness is shown by the horizontal dashed line at $f \delta_{0} / u_{e}=0.008$. Discontinuities in the WPS are caused by the plotting style, since WPS are down-sampled in time due to memory constraints (global features do not change)

FSS $\rightarrow$ RSS transition occurs at similar NPRs and that global features of the pressure signatures are visually identical (Baars et al, 2011).

Fig. 16 presents the sweep of the incipient separation shock foot's intermittent region over location $x_{8}$ for all three ramps; WPS are shown in sub-figures a, $\mathrm{c}$ and e with corresponding normalized pressure timeseries $p\left(x_{8}, t\right) / p_{a}$ being shown in sub-figures $\mathrm{b}, \mathrm{d}$ and $\mathrm{f}$, respectively. Beginning with the pressure time series, they are shown to transition from experiencing a broad spectrum of high energy unsteadiness in the $1^{\text {st }}$ separation bubble to relatively low-energetic activity, superposed sparsely distributed and large amplitude spikes associated with an attached boundary layer and a passing shock. The raw time-series demonstrate how a decreasing ramp rate causes more shock crossings during the sweep, which is expected, given the longer amount of time the transducer is exposed to the intermittent region (note that the abscissa on each plot does not span a similar time range).

As for the pre-multiplied Mexican hat WPS shown in sub-figures a, c and e, they are being presented with non-dimensional frequency on the right ordinate, and non-dimensional time on the top abscissa. Contour levels represent the pre-multiplied spectra, and are presented in a logarithmic fashion to account for the large range of energy scales. The cone of influence of the Mexican hat wavelet is also shown to illustrate the temporal domain influenced by each wavelet scale. When the intermittent region sweeps over this location, most of the energy is shown to reside with frequencies centered around $150 \mathrm{~Hz}$ (this corresponds to a non-dimensional frequency of $f \delta_{0} / u_{e} \approx 0.0005$ ).

A quantitative comparison between the peak-frequency of the non-stationary shock motion unsteadiness with what was observed during stationary conditions (Fig. 12b) is now made. Time-averaged WPS are evaluated over five non-overlapping windows in the intermittent region. These are denoted as I1 through I5 in Fig. 16e$\mathrm{f}$ and span only $0.02 \mathrm{~s}$ each. The individual windowaveraged WPS are shown in Fig. 17, as well as the resultant trend that one obtains by averaging over all windows (dashed black line). The ordinate on the lefthand side has been non-dimensionalized by the standard of deviation of the stationary pressure time-series at location $x_{8}$, as was used in Fig. 12b. The factor of two is incorporated as we are comparing these timeaveraged WPS to the one-sided, pre-multiplied PSD in Fig. $12 \mathrm{~b} ; 2 \bar{E} f / \sigma^{2}=G f / \sigma^{2}$ would have to hold if the signal used to generate $\bar{E}\left(x_{8} ; f\right)$ would have been the non-transient, stationary signal. Given that the individual spectra corresponding to windows I1 to I5 are generated over a short temporal window length, it is not surprising that each individual spectrum looks different. That is, the features that appear in the window average spectra -centered around $150 \mathrm{~Hz}$ and 1,500 Hz-do not appear periodically in time. This is the case for most random stationary data, which necessitates ensemble averaging. For now, it is assumed that the average over all of the individual window spectra (dashed line in 

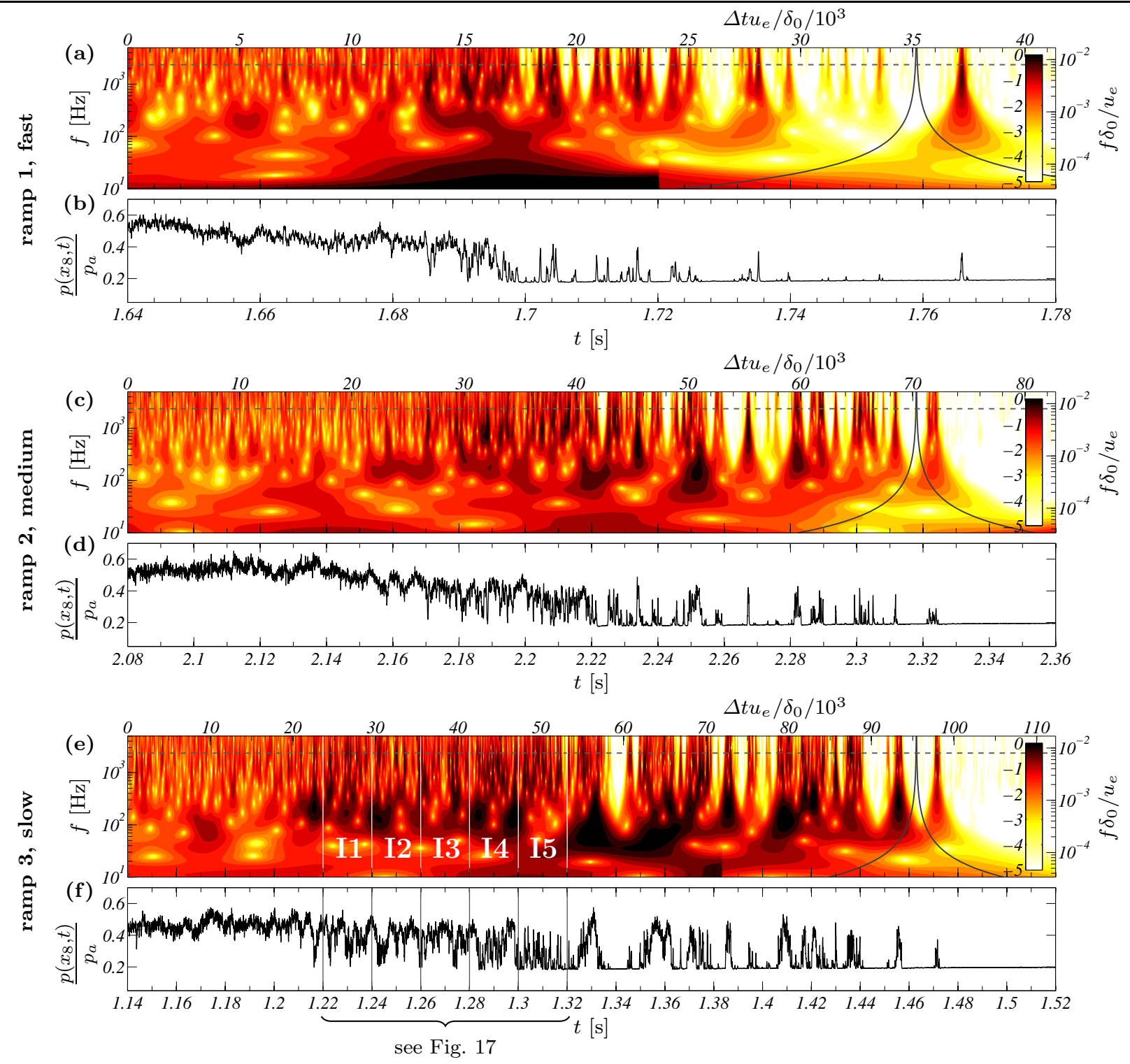

Fig. 16 a,c,e Pre-multiplied Mexican hat WPS of the fluctuating wall pressure at location $x_{8}$ with contour levels defined as $\log _{10}\left(E\left(x_{8} ; f, t\right) \cdot f\right)$ in $\left[\mathrm{psia}^{2}\right]$ for a ramp 1, b ramp 2 and $\mathbf{c}$ ramp 3. b,d,f Raw pressure time-series $p\left(x_{8}, t\right) / p_{a}$ synchronously shown with the WPS. Normalized time is shown on the top abscissa of the WPS plot, and normalized frequency is shown on the right ordinate; values used for normalization are provided in table 1 . The lower threshold for stationary low-frequency shock motion unsteadiness is shown by the horizontal dashed line at $f \delta_{0} / u_{e}=0.008$

Fig. 17) is converged and representative of the shock motion unsteadiness during non-stationary operation. Ideally, one would repeat the analysis for many experimental runs and check that the spectrum is indeed converged and representative, however, this is practically challenging and costly. A comparison between the obtained wall pressure spectrum at location $x_{8}$ during stationary operation and the spectrum obtained during non-stationary operation is now shown in Fig. 18. When assembling this figure, the amplitude of the PSD of the stationary case was divided by a factor of 7.5. Also, the non-stationary spectrum is obtained via the waveletbased time-frequency analysis and thus involved an im- plicit scale-filtering that makes it visually smooth. It can be seen how the energy content is similar for the stationary and non-stationary scenarios. These data suggest that the low-frequency shock motions, reflected in the spectra by a peak at $150 \mathrm{~Hz}$, and the loweramplitude fluctuations around $1,500 \mathrm{~Hz}$, are present for both scenarios. Aside from the energized frequency bands, it is revealed that the energy amplitude during the non-stationary operation is reduced by a factor of 7.5 , since the spectra collapse on the figure, where the stationary one is divided by 7.5 before plotting. Thus, this shows that during transient operation the pressure fluctuations associated with the low-frequency 


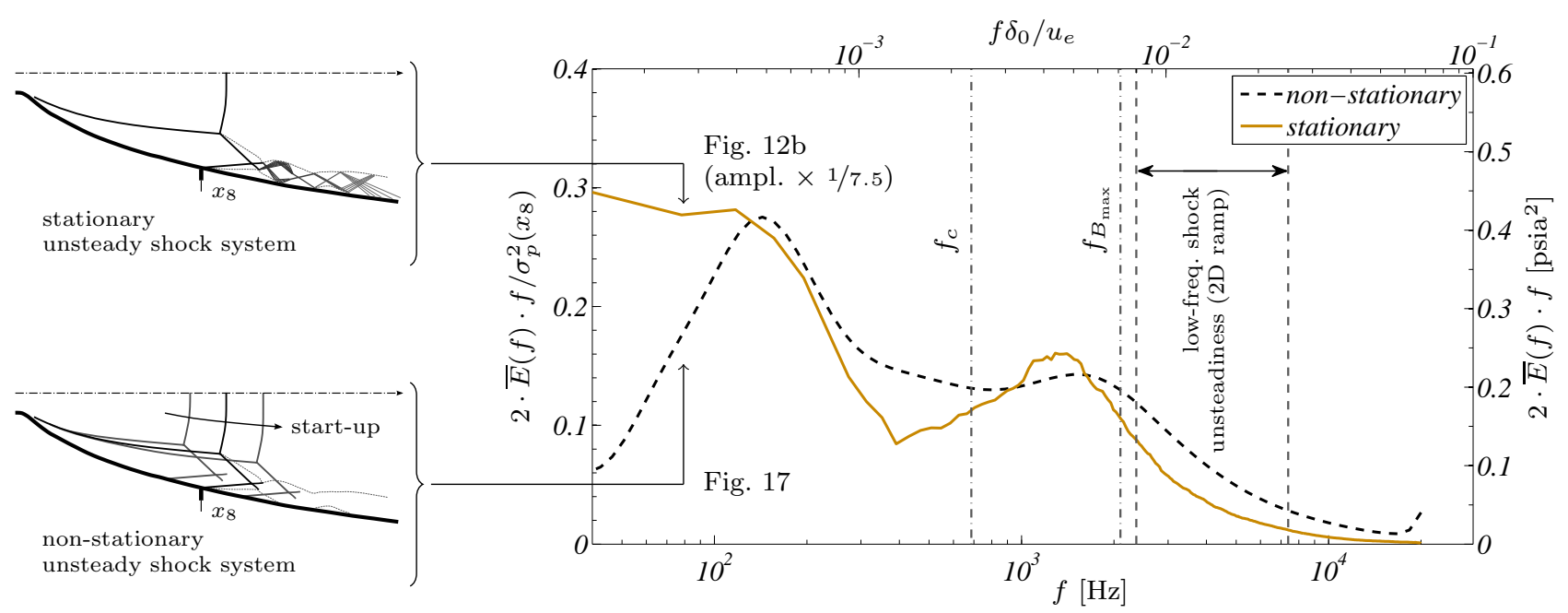

Fig. 18 Comparing the PSD of the dynamic wall pressure at location $x_{8}$ during stationary operation of the nozzle (obtained via conventional Fourier analysis of the stationary time-series) and non-stationary operation of the nozzle (obtained via wavelet-based time-frequency analysis); the spectra are taken from Figs. 12b and 17, respectively

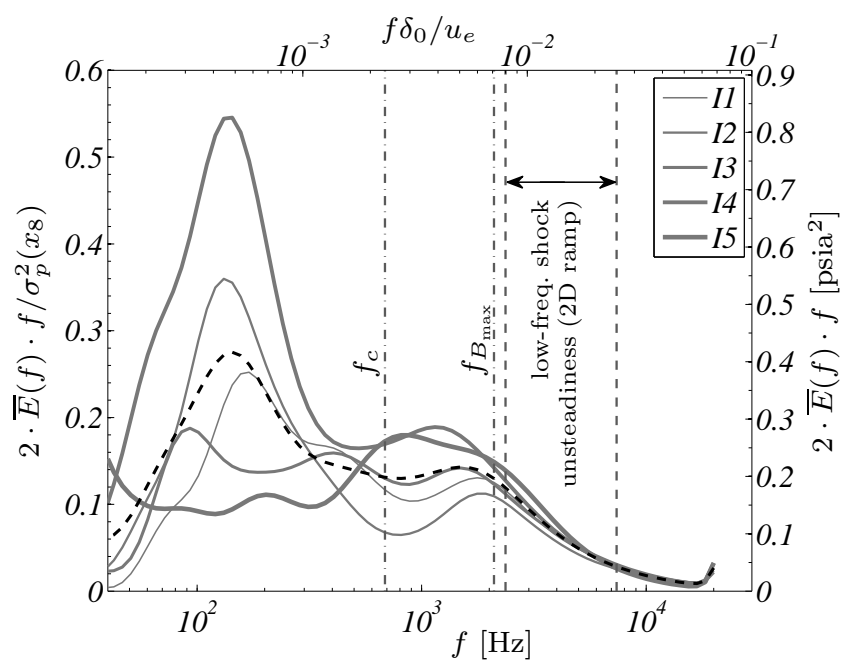

Fig. 17 Local time-averaged Mexican hat WPS when the shock sweeps past the transducer (the intermittent region sweeps over $x_{8}$ ), windows of averaging have a temporal range of $\Delta t=0.02 \mathrm{~s}$ or $\Delta t u_{e} / \delta_{0}=5,900$ and $\sigma_{p}^{2}\left(x_{8}\right)=1.514 \mathrm{psia}^{2}$. The dashed black line is the average over all windows

shock motion unsteadiness are damped. Figs. 17 and 18 and the discussion above are only valid for the slowest ramp. Comparing this ramp to ramps 1 and 2 in Figs. 16a and 16c, respectively, the signal comprises less energy in the intermittent region. This demonstrates how the amount of unsteadiness that the system is exposed to during transient SWBLI depends significantly on the transient rate. The findings suggest that the local wall pressure unsteadiness becomes less pronounced when transient rates are increased. To summarize, during ramp 3, where the non-dimensional ramp rate at the moment that the intermittent region passes location $x_{8}$ is $\left(L / a^{*}\right) d \mathrm{NPR} / d t=0.7 \cdot 10^{-2}$, the energy is reduced by a factor of 7.5 compared to the stationary operating scenario, while for ramp 1 and 2 (non-dimensional ramp rates of $3.1 \cdot 10^{-2}$ and $1.5 \cdot 10^{-2}$, respectively) the amplitude decrease can not be defined since similar energetic features can not be identified. A future parametric study, comprising numerous repeated trials and various ramp rates would provide for statistically converged results capable of confirming the hypothesis that the energetic features of low-frequency shock motion unsteadiness reduce strongly in amplitude for increasing ramp rate.

\subsection{Global influence of transient unsteadiness}

In this last section, the integrated unsteadiness, or global unsteadiness, exerted by the internal flow topology on part of the nozzle's interior surface is studied for all three transients. The focus is to establish a single metric that is capable of quantifying the global unsteadiness acting on the system during transient operations. Earlier work by Baars and Tinney (2013) investigated the transient unsteadiness at two stream wise positions by taking the azimuthal three-dimensionality into account. Here, we only focus on the unsteadiness along the stream wise array of transducers in the aft section of the nozzle. It is assumed that three-dimensional effects are inherently included. We begin by presenting the unsteady behaviour at one stream wise location $\left(x_{10}\right)$ in Fig. 19. The transient NPR range is confined to $\mathrm{NPR}=[10,50]$ (for both start-up and shut-down). Start-up is denoted by 'START' with $\Delta T$ corresponding to the absolute time of the start-up through this NPR range. As for shut-down, denoted by 'STOP', time pro- 

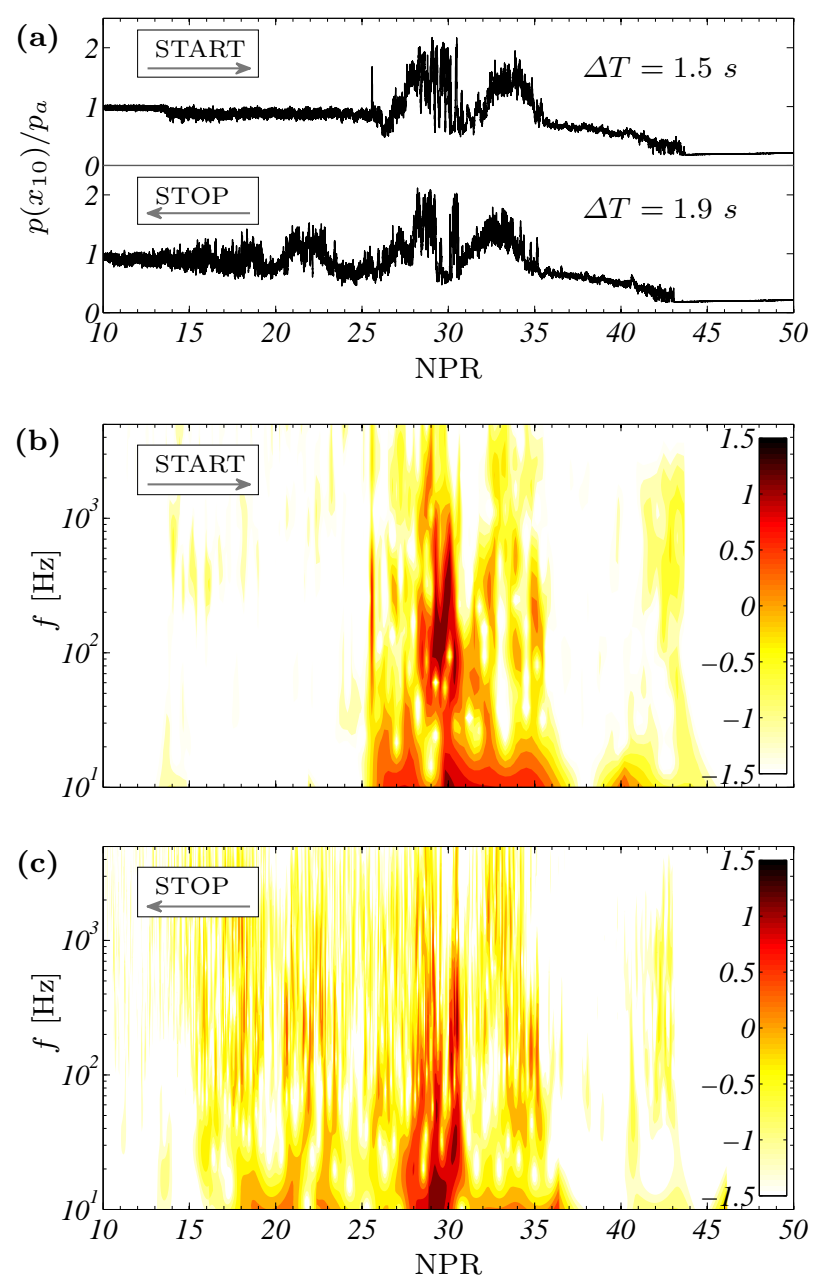

Fig. 19 a raw pressure time traces for ramp 1 as function of NPR for the start-up (START) and shut-down (STOP); $\Delta T$ is the duration associated with the NPR range. b,c Local wavelet spectra, plotted as $\log _{10}\left(E\left(x_{10} ; f, t\right) \cdot f\right)$ in $\left[\mathrm{psia}^{2}\right]$, for the start-up and shut-down

gresses from right to left, as indicated by the arrow. The normalized pressure time-series and the pre-multiplied Mexican hat WPS for both start-up and shut-down are also shown. The metric that we will use to characterize this global unsteadiness is obtained by simply integrating the wavelet spectrum, denoted as the total wavelet energy, according to,

$E_{W}\left(x_{j}\right)=2 \int_{10}^{f_{\max }}\left\{\frac{1}{T} \int_{T} E\left(x_{j} ; f, t\right) d t\right\} d f$,

where $f_{\max }=5 \mathrm{kHz}$. This metric represents the total energy exerted on transducer $x_{j}$ per unit time and can be interpreted as the variance of the transient signal over the frequency range $10 \mathrm{~Hz}<f<5 \mathrm{kHz}$; a factor of two has been included since the wavelet spectrum is one-sided. Fig. 14 shows how the four aft locations $\left(x_{9}-x_{12}\right)$ experience most of the RSS shock structure unsteadiness. For this reason the total wavelet ener-
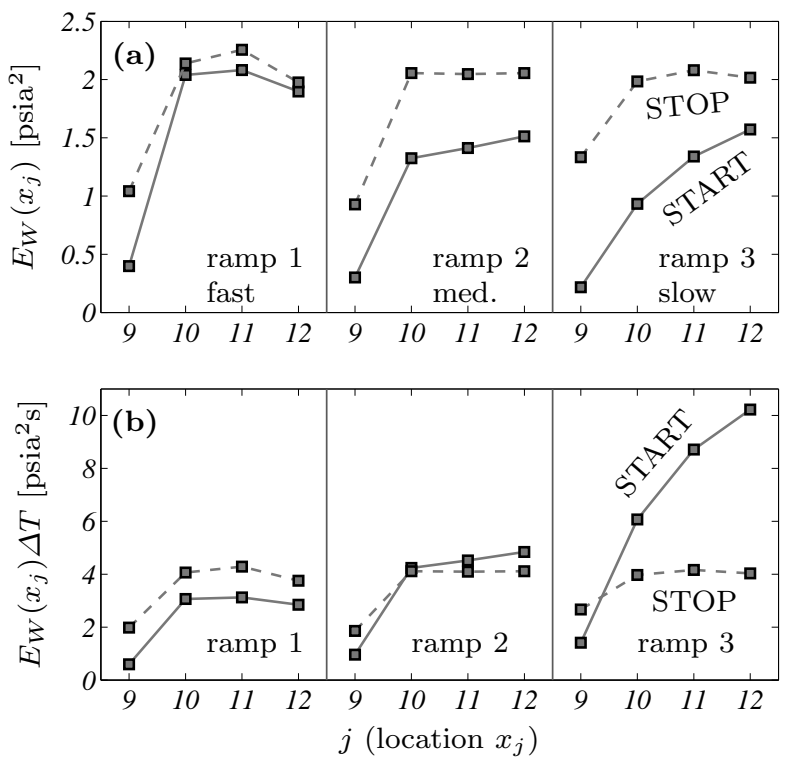

Fig. 20 a Total wavelet energy $E_{w}$ during start-up and shutdown at the four aft locations in the axisymmetric nozzle. $\mathbf{b}$ Exposed energy to the global system $E_{W} \Delta T$, where $\Delta T$ is the duration of the ramp indicated in Fig. 19

gies $E_{W}\left(x_{j}\right)$ are calculated at these locations for each ramp, the results of which are visualized in Fig. 20a. The trends indicate that the global unsteadiness per unit time during start-up decreases when the transient start-up rates are slower (ramp $1 \rightarrow 3$ ). Earlier observations in Fig. 16, which focused on the localized SWBLI unsteadiness, showed how wall pressure unsteadiness increased with decreasing ramp rate. Here, it is apparent that trends for the localized unsteadiness do not translate one-to-one to the global system. That is, the global system is exposed to unsteadiness of the entire RSS shock train, while the localized unsteadiness was analysed for the incipient separation shock foot at one particular stream wise location upstream $\left(x_{8}\right.$, see also Fig. 14b). An increase in unsteadiness nearer the nozzle exit plane was also observed by Johnson and Papamoschou (2010) and can be explained by an increase in the shock strength when moving aft.

Concerning the three shut-downs with similar ramp rates, a small variation in $E_{W}$ for ramps 1 to 3 is attributed to the natural variations that are expected with each trial. The three sub figures in Fig. 20a show that the unsteadiness per unit time of the shut-down transients are higher than those of the start-ups. However, as mentioned previously, all shut-down ramps were of a similar rate, whereas the start-up $d \mathrm{NPR} / d t$ decreased for ramp $1 \rightarrow 2 \rightarrow 3$. Note that for ramp 1 , where the $d \mathrm{NPR} / d t$ for start- and shut-down are nearly identical, the magnitudes of unsteadiness per unit time for $x_{10}, x_{11}$ and $x_{12}$ (i.e. those locations that experience 
a significant amount of RSS during start-up) are similar. It is conjectured that the differences in magnitude of unsteadiness per unit time are probably more related to differences in ramp rate than in ramp direction and that if the shut-down $d \mathrm{NPR} / d t$ rates were to be decreased the per unit time unsteadiness at each location would decrease.

Now we quantify the total energy that the system is exposed to; displayed in Fig. 20b by multiplying the wavelet energy by the duration of the ramp, $\Delta T$ (the time that the system is exposed to SWBLI unsteadiness). These findings are insightful to those interested in performing life cycle analyses. Glancing at ramp 2 for example, it is clear that the wavelet energy during shutdown is higher (Fig. 20a) than start-up, thus demonstrating how the shut-down process produces more violent SWBLI activity. However, due to the shorter exposure time during shut-down $(1.9 \mathrm{~s})$ as opposed to startup $(3.2 \mathrm{~s})$, the total energy that the system is exposed to during this start-up and shut-down is roughly equal (Fig. 20b).

\section{Summary and conclusions}

We have surveyed the low-frequency wall pressure unsteadiness of the internal flow of a large area-ratio supersonic nozzle $\left(M_{d}=5.58\right)$ during overexpanded operations. As has been observed elsewhere, multiple shock wave / boundary layer interactions, associated with turbulent boundary layer separations were shown to reside within the diverging section of the nozzle and comprised low-frequency shock motion unsteadiness.

Two different operating scenarios of the nozzle were considered. The first focused on low-frequency shock motion unsteadiness during a fixed (stationary) pressure ratio of 28.7. The incipient shock-induced separation being considered was part of an annular shock train that is classified as a restricted shock separation. The stationary low-frequency unsteadiness of the shock foot was shown to be centred around a non-dimensional shock-zero crossing frequency of $S t_{\delta}=f_{c} \delta_{0} / u_{e} \approx 0.004$ at a position in the intermittent region where the intermittency was $\gamma=64.4 \%$. Furthermore, wall pressure unsteadiness in the separated regions downstream of the interaction decreased when the shock moved downstream, and vice versa. This was found through an averaging technique conditioned on upstream and downstream shock motions, respectively. The out-of-phase pressure fluctuations are indicative of a relatively long intermittent region, as the phenomenon can be explained by translating the static wall pressure profile back and forth with the shock foot's motion. This long intermittent region is plausible since the shock foot is not an- chored by geometric features, as is the case with compression ramps and blunt fins for example.

The second operating scenario focused on transient nozzle pressure ratios with an emphasis on nozzle startups and shut-downs. A wavelet based time-frequency analyses was employed to characterize the spectral content of the wall pressure fluctuations during sweeping of the shock foot's intermittent region. Time-frequency analyses of the wall pressure at the same location at which the stationary unsteadiness was analysed, revealed how the frequency content and energetic amplitude compared between unsteady fluctuations that are induced by either a stationary or non-stationary shock system. Here we only focussed on the incipient separation shock of a RSS flow structure near location $x_{8}$. For a relatively slow start-up transient, the frequency content was similar to the stationary case while the amplitude of the unsteadiness was reduced by a factor of seven. The transient rate of start-up is also shown to have a significant influence on the spectral features of the low-frequency shock motion unsteadiness since similar frequency content was not observed for the faster startups. Therefore, the low-frequency shock motions and unsteady pressure fluctuations are not superposed on a transient mean in an uncoupled sense.

Acknowledgements Funding for this effort was graciously provided by a grant from the Air Force Office of Scientific Research (FA9550-11-1-0203) with Dr. J. Schmisseur as technical monitor, as well as the NASA Engineering and Safety Center. A great portion of this work was written while WJB was a Post-Doctoral Research Fellow at the University of Melbourne, Australia and being supported by funds of the Australian Research Council.

\section{References}

Addison PS (2002) The illustrated wavelet transform handbook. Taylor \& Francis, New York

Baars WJ, Tinney CE (2013) Transient wall pressures in an overexpanded and large area ratio nozzle. Exp Fluids $54: 1468$

Baars WJ, Tinney CE, Ruf JH (2011) Time-frequency analysis of rocket nozzle wall pressures during start-up transients. J Phys Conf Ser 318(9):1-10

Baars WJ, Tinney CE, Ruf JH, Brown AM, McDaniels DM (2012) Wall pressure unsteadiness and side loads in overexpanded rocket nozzles. AIAA J 50(1):61-73

Babinsky H, Harvey JK (2011) Shock wave-boundary-layer interactions. Cambridge University Press, New York

Beresh SJ, Clemens NT, Dolling DS (2002) Relationship between upstream turbulent boundary-layer velocity fluctuations and separation shock unsteadiness. AIAA J 40(12):2412-2422

Clemens NT, Narayanaswamy V (2014) Low-frequency unsteadiness of shock wave/turbulent boundary layer interactions. Annu Rev Fluid Mech 46:469-492 
Cohen L (1989) Time-frequency distributions - a review. Proc IEEE 77(7):941-981

Das D (1987) A numerical study of turbulent separated flows. ASME FED 51:85-90

Dolling DS (2001) Fifty years of shock-wave/boundary-layer interaction research: What next? AIAA J 39(8):1517-1531

Dolling DS, Brusniak L (1989) Separation shock motion in fin, cylinder, and compression ramp - induced turbulent interactions. AIAA J 27(6):734-742

Dolling DS, Or CT (1985) Unsteadiness of the shock wave structure in attached and separated compression ramp flows. Exp Fluids 3(4):24-32

Donald BW, Baars WJ, Tinney CE, Ruf JH (2014) Sound produced by large area-ratio nozzles during fixed and transient operations. AIAA J 52(7):1474-1485

Dupont P, Haddad C, Debiève JF (2006) Space and time organization in a shock-induced separated boundary layer. J Fluid Mech 559:255-277

Erengil ME, Dolling DS (1991) Unsteady wave structure near separation in a Mach 5 compression ramp interaction. AIAA J 29(5):728-735

Farge M (1992) Wavelet transforms and their application to turbulence. Annu Rev Fluid Mech 24:395-457

Fernholz HH, Warnack D (1998) The effects of a favourable pressure gradient and of the Reynolds number on an incompressible axisymmetric turbulent boundary layer. Part 1. The turbulent boundary layer. J Fluid Mech 359:329356

Frey M, Hagemann G (2000) Restricted shock separation in rocket nozzles. J Prop Power 16(3):478-484

Gallagher JA, McLaughlin DK (1981) Experiments on the nonlinear characteristics of noise propagation from low and moderate Reynolds number supersonic jets. In: 7th AIAA aeroacoustics conf, Palo Alto, CA, AIAA 1981-2041

Ganapathisubramani B, Clemens NT, Dolling DS (2009) Low-frequency dynamics of shock-induced separation in a compression ramp interaction. J Fluid Mech 636:397-425

Gonsalez JC, Dolling DS (1993) Correlation of interaction sweepback effects on the dynamics of shock-induced turbulent separation. In: 31st AIAA Aerospace Sciences Meeting, Reno, NV, AIAA 1993-0776

Humble RA, Scarano F, van Oudheusden BW (2009) Unsteady aspects of an incident shock wave/turbulent boundary layer interaction. J Fluid Mech 635:47-74

Johnson AD, Papamoschou D (2010) Instability of shockinduced nozzle flow separation. Phys Fluids 22, 016102

Luke E (2007) On robust and accurate arbitrary polytope cfd solvers. In: 18th AIAA computational fluid dynamics conf, Miami, FL, AIAA 2007-3956

Luke E, Cinella P (2007) Numerical simulations of mixtures of fluids using upwind algorithms. Computers and Fluids 36(10):1547-1566
Menter FR (1992) Influence of freestream values on $\kappa-\omega$ turbulence model predictions. AIAA J 30(6):1657-1659

Menter FR (1994) Two-equation eddy-viscosity turbulence models for engineering applications. AIAA J 32(8):15981605

Nave LH, Coffey GH (1973) Sea level side loads in high-arearatio rocket engines. In: 9th AIAA Prop. Conf, Las Vegas

Nguyen AT, Deniau H, Girard S, Alziary de Roquefort T (2003) Unsteadiness of flow separation and end-effects regime in a thrust-optimized contour rocket nozzle. Flow, Turb Comb 71:161-181

Olson BJ, Lele SK (2013) A mechanism for unsteady separation in over-expanded nozzle flow. Phys Fluids 25, 110809

Piponniau S, Dussauge JP, Debiève JF, Dupont P (2009) A simple model for low-frequency unsteadiness in shockinduced separation. J Fluid Mech 629:87-108

Plotkin KJ (1975) Shock wave oscillation driven by turbulent boundary-layer fluctuations. AIAA J 13(8):1036-1040

Poggie J, Smits AJ (1997) Wavelet analysis of wall-pressure fluctuations in a supersonic blunt-fin flow. AIAA J 35(10):1597-1603

Priebe S, Martín MP (2012) Low-frequency unsteadiness in shock wave-turbulent boundary layer interaction. J Fluid Mech 699:1-49

Ruf JH, McDaniels DM, Brown AM (2009) Nozzle side load testing and analysis at MSFC. In: 45th AIAA joint prop conf, Denver, CO, AIAA 2009-4856

Ruf JH, McDaniels DM, Brown AM (2010) Cold flow test results for nozzle side loads for J-2X and SSME test articles. In: 57th JANNAF prop meeting, Colorado Springs, CO

Sarkar S, Lakshmanan B (1991) Applicaiton of reynolds stress turbulence model to the compressible shear layer. AIAA J 29(5):743-749

Smits AJ, Dussauge JP (2006) Turbulent shear layers in supersonic flow. Springer, New York

Thomas FO, Putman CM, Chu HC (1994) On the mechanism of unsteady shock oscillation in shock wave/turbulent boundary layer interactions. Exp Fluids (18):69-81

Verma SB, Haidn O (2014) Unsteady shock motions in an over-expanded parabolic rocket nozzle. Aerospace Science and Technology 39:48-71

Wang TS (2009) Transient three-dimensional startup side load analysis of a regeneratively cooled nozzle. Shock Waves 19(3):251-264

Wilcox DC (1998) Turbulence modeling for CFD. DCW Industries

Wu M, Martín MP (2008) Analysis of shock motion in shockwave and turbulent boundary layer interaction using direct numerical simulation data. J Fluid Mech 594:71-83

Zaman KBMQ, Dahl MD, Bencic TJ, Loh CY (2002) Investigation of a 'transonic resonance' with convergent-divergent nozzles. J Fluid Mech 463 


\section{University Library}

\section{- M M I N E R VA A gateway to Melbourne's research publications}

Minerva Access is the Institutional Repository of The University of Melbourne

Author/s:

BAARS, $W$

Title:

Non-stationary shock motion unsteadiness in an axisymmetric geometry with pressure gradient

Date:

2015

Citation:

BAARS, W. (2015). Non-stationary shock motion unsteadiness in an axisymmetric geometry with pressure gradient. Experiments in Fluids: experimental methods and their applications to fluid flow, 56 (5), https://doi.org/10.1007/s00348-015-1958-y.

Persistent Link:

http://hdl.handle.net/11343/55183 